Journal of Intelligent Material Systems \& Structures

\title{
Optimal shunt parameters for maximising wave attenuation with periodic piezoelectric patches
}

\begin{tabular}{|c|c|}
\hline Journal: & Journal of Intelligent Material Systems and Structures \\
\hline Manuscript ID: & Draft \\
\hline Manuscript Types: & Original Article \\
\hline Date Submitted by the Author: & $\mathrm{n} / \mathrm{a}$ \\
\hline Complete List of Authors: & $\begin{array}{l}\text { Lee, Junyi; Imperial College London, Mechanical Engineering Department } \\
\text { Balint, Daniel; Imperial College London, Mechanical Engineering } \\
\text { Department }\end{array}$ \\
\hline Keyword: & Control, Piezoelectric, Optimization \\
\hline Abstract: & $\begin{array}{l}\text { Elastic metamaterials, which have huge potential in wave guiding and } \\
\text { attenuation applications, can be built from structures with periodic } \\
\text { piezoelectric patch arrays. Passive shunts offer the benefits of simplicity } \\
\text { and low cost. In this paper, the effects of the magnitude and phase angle } \\
\text { of the shunt impedance on the attenuation constant of a beam with } \\
\text { periodic piezoelectric patch arrays were studied in order to determine the } \\
\text { optimal shunt that produces the widest and most effective band gaps. The } \\
\text { attenuation constants were found to be large when the phase angle is } n / 2 \\
\text { rad and when the magnitude decreases exponentially with the excitation } \\
\text { frequency. This corresponds to a negative capacitance circuit, which is the } \\
\text { optimal shunt for such systems. The attenuation constant of the system } \\
\text { reduces significantly when the impedance deviates from the optimal value } \\
\text { suggesting other circuits are less effective. The impedance and band } \\
\text { structure of resistive-inductive (R-L), negative capacitance and resistive } \\
\text { shunts were investigated. As expected, the negative capacitance circuit } \\
\text { produces a large band gap, while the R-L circuit only produces a band gap } \\
\text { around its natural frequency. The transmissibilities of a finite system with } \\
\text { these circuits demonstrated that vibration transmissions are low within the } \\
\text { band gaps. }\end{array}$ \\
\hline \multicolumn{2}{|c|}{$\begin{array}{l}\text { Note: The following files were submitted by the author for peer review, but cannot be converted to } \\
\text { PDF. You must view these files (e.g. movies) online. }\end{array}$} \\
\hline SageV.bst & \\
\hline
\end{tabular}




1
2
3
4
5
6
7
8
9
10
11
12
13
14
15
16
17
18
19
20
21
22
23
24
25
26
27
28
29
30
31
32
33
34
35
36
37
38
39
40
41
42
43
44
45
46
47
48
60
59
50
52
53
55
50
32 34 35 36 38 39 40 41 43 44 45 


\title{
Optimal shunt parameters for maximising wave attenuation with periodic piezoelectric patches
}

\author{
Lee Junyi ${ }^{1}$ and Daniel Stephen Balint ${ }^{1}$
}

\begin{abstract}
Elastic metamaterials, which have huge potential in wave guiding and attenuation applications, can be built from structures with periodic piezoelectric patch arrays. Passive shunts offer the benefits of simplicity and low cost. In this paper, the effects of the magnitude and phase angle of the shunt impedance on the attenuation constant of a beam with periodic piezoelectric patch arrays were studied in order to determine the optimal shunt that produces the widest and most effective band gaps. The attenuation constants were found to be large when the phase angle is $\frac{\pi}{2}$ rad and when the magnitude decreases exponentially with the excitation frequency. This corresponds to a negative capacitance circuit, which is the optimal shunt for such systems. The attenuation constant of the system reduces significantly when the impedance deviates from the optimal value suggesting other circuits are less effective. The impedance and band structure of resistive-inductive (R-L), negative capacitance and resistive shunts were investigated. As expected, the negative capacitance circuit produces a large band gap, while the R-L circuit only produces a band gap around its natural frequency. The transmissibilities of a finite system with these circuits demonstrated that vibration transmissions are low within the band gaps.
\end{abstract}

\section{Keywords}

Band gap, Bloch wave theory, band structure, phononic crystals, elastic metamaterials, periodic structures, piezoelectric patches, passive shunt

\section{Introduction}

Piezoelectric patches have been widely considered in vibration damping and control applications in structures as they have the ability to convert electrical energy to mechanical energy and vice versa (Hagood and von Flotow (1991)). This allows the implementation of a variety of control strategies to damp vibrations. The passive shunt configuration, in which the piezoelectric patch is connected to a passive circuit, is considered to be one of the simplest control system because it avoids the need of complex amplifiers and sensing equipment (Hagood and von Flotow (1991)) required in active control strategies. Several configurations of passive piezoelectric patches have been proposed, for example the resistive-inductive (R-L) circuit (Hagood and von Flotow (1991)) that acts as a dynamic vibration absorber reducing vibrations at the tuned frequencies. Later studies have introduced other circuits for shunted piezoelectric patches that makes use of different concepts, including multiple resonating circuits ( $\mathrm{Wu}$ and McDonnell (1996); Behrens et al. (2003)) and negative capacitance shunts (Beck (2012)).

With increasing interest in elastic metamaterials, piezoelectric patches arranged in periodic arrays (Thorp et al. (2001); Spadoni et al. (2009); Airoldi and Ruzzene (2011); Chen et al. (2013a,b); Casadei et al. (2012)) have been studied in order to create elastic metamaterials with tunable band gaps, which is a desired property for this class of materials (Hussein et al. (2014)). Unlike conventional configurations for vibration attenuation, the periodic shunted piezoelectric patch arrangement creates band gaps, which are frequency ranges in which wave propagation cannot occur, to attenuate elastic waves without targeting a given mode of vibration (Spadoni et al. (2009)). This strategy results in broadband vibration attenuation as opposed to damping at specific frequencies typical of conventional systems. Two common circuits used to introduce band gaps are the R-L circuit (Spadoni et al. (2009); Airoldi and Ruzzene (2011); Chen et al. (2013a)) and the negative capacitance circuit (Tateo et al. (2014); Beck et al. (2012)). For the R-L circuit, the band gaps are created via the local resonant mechanism in elastic metamaterials proposed by (Liu et al. (2000)) with the periodic piezoelectric patches acting as the tuned resonators. Negative capacitance shunts on the other hand have been known to reduce vibrations over a large range of frequencies. This is done by tuning the capacitance of the shunt to values close to the capacitance of the shunt so that the effective stiffness of the patch defined by Eq. (1) (Hagood and von Flotow (1991)) is sufficiently large to prevent motion from passing through. Hence, by using a periodic piezoelectric patch with negative capacitance, large mechanical impedance mismatch can be achieved to create wide band-gaps for vibration attenuation.

\footnotetext{
${ }^{1}$ Imperial College London, UK
}

\section{Corresponding author:}

Lee Junyi, Imperial College London, Mechanical Engineering Department, South Kensington Campus, London SW7 2AZ.

Email: junyi.lee108@imperial.ac.uk 


$$
E_{p}^{S U}(\omega)=E_{p}^{D}\left(1-\frac{k_{31}^{2}}{1+i \omega C_{p}^{\varepsilon} Z^{S U}(\omega)}\right)
$$

where $E_{p}^{S U}$ is the effective Young's Modulus of the piezoelectric patch, $E_{p}^{D}$ is the Young's Modulus of the piezoelectric patch at constant electrical displacement, $\omega$ is the excitation frequency, $k_{31}$ is the electro-mechanical coupling coefficient, $C_{p}^{\varepsilon}$ is the capacitance and $Z^{S U}$ is the impedance of the shunt.

Since the shunt parameters, which are the inductance, $L$, capacitance, $C$, and resistance, $R$, will affect the vibration attenuation performance of the periodic arrays, many studies (Thorp et al. (2001); Spadoni et al. (2009); Chen et al. (2013a)) have analysed the effects of these parameters on the band gap width, location, and magnitude of the attenuation constants for plates or beams with periodic piezoelectric patches. However, in those studies, the shunting circuit was selected prior to the analysis of the effects of the parameters on the band gaps. This limits the analyses to the selected circuit while neglecting the possibility that other circuit configurations might have better performance. Regardless of the selected circuit, the electrical impedance of the passive shunt, $Z^{S U}$, can be expressed in terms of only the magnitude, $a$, and the phase angle, $\phi$, using Eq. (2).

$$
Z^{S U}=a \cdot e^{i \phi}
$$

Therefore, the wave attenuation characteristics of all possible passive shunts can be studied by analysing only $a$ and $\phi$. Hence, a map of the attenuation constant over a range of frequencies and shunt impedances will provide valuable information to the design of shunts for elastic wave attenuation using periodic shunted piezoelectric patches. In this study, the attenuation constants for a beam with periodic piezoelectric patches will be calculated over a range of magnitudes and phase angles of the shunt impedance. The map of the attenuation constants will then be analysed to devise design strategies for the passive shunts.

This paper is organised as follows. Firstly, the beam and piezoelectric parameters, the models for the beam and piezoelectric patches, and the method used to calculate the attenuation constants will be described. After that, a map, which is a volume visualisation of the attenuation constants with respect to excitation frequency, magnitude and phase angle of shunt impedances will be presented. Then, the trends observed in the map and a suitable design strategy for the passive shunt will be discussed. Additionally, the band structure of the R-L and negative capacitance shunts will be discussed with regards to the map, and the optimal shunt configuration will be found.

\section{Description of beam and models}

\section{Beam geometry}

An infinite beam with piezoelectric patches, as seen in Fig. 1, was studied. A beam was selected instead of a plate mainly because the elastic wave in a beam only travels in a single direction, which simplifies the study as only the attenuation constant in one dimension needs to be considered. Maps for plates that have additional dimensions can be found using the same analysis and similar trends are expected to be found for plates with similar configurations.

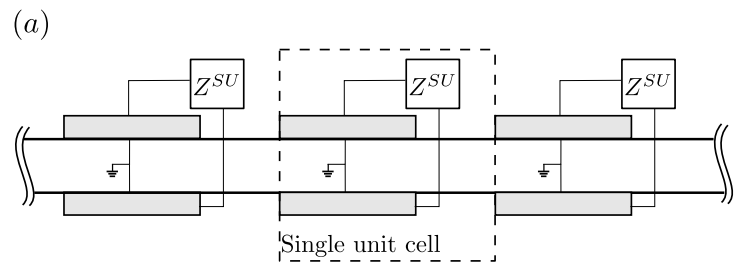

(b)

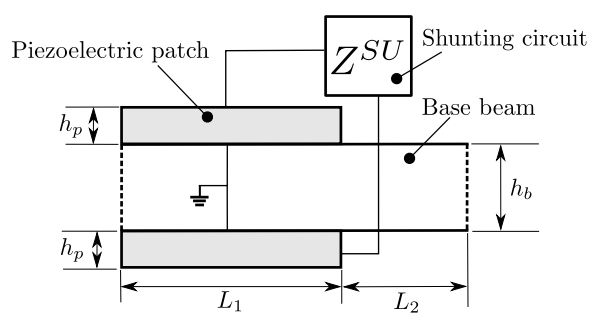

Figure 1. Infinite beam with periodic piezoelectric patch (a) A structure with infinitely repeating unit cells (b) A single unit cell.

The properties of the piezoelectric patch and beam used in this study are listed in Table 1 while the geometry of the unit cell is listed in Table 2. The shunting circuits for the top and bottom piezoelectric patch were set to be identical.

\section{Beam and piezoelectric model}

The model proposed by Ha (2001) was used to model the beam and piezoelectric patch in this study. This model was selected mainly because the Timoshenko beam model, which takes into account rotational inertia, results in more accurate predictions, especially at higher frequencies. Additionally, the model proposed by Ha (2001) allows the piezoelectric patch to be used as both sensor and actuator, which allows the same model to be used in future studies involving active control.

In the Ha (2001) model, the dynamic behaviour of the piezoelectric patch and the beam, using the sign convention shown in Fig. 2, is described by the transfer matrix in Eq. (3).

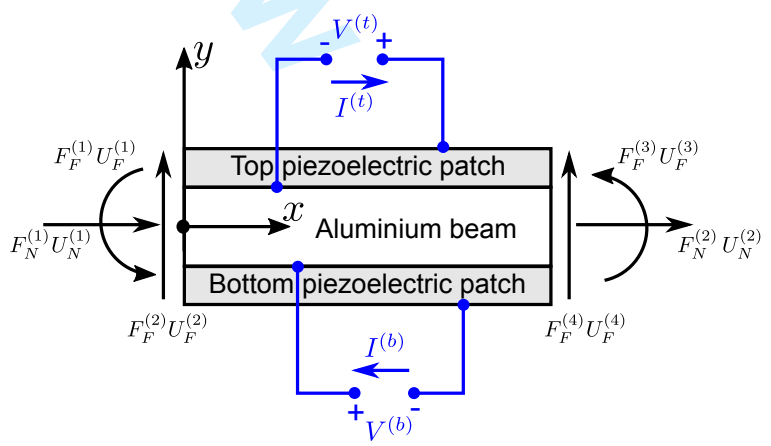

Figure 2. Piezoelectric patch and beam model.

$$
\left[\begin{array}{c}
\mathbf{F}_{N} \\
\mathbf{F}_{F} \\
\mathbf{I}
\end{array}\right]=\mathbf{D}\left[\begin{array}{c}
\mathbf{U}_{N} \\
\mathbf{U}_{F} \\
\mathbf{V}
\end{array}\right]
$$

where the terms are described by Eqs. (4) to (10); the terms within the dynamic stiffness matrix, D, in Eq. (4) are listed 
Table 1. Properties of piezoelectric patch and base beam.

\begin{tabular}{lcc}
\hline Property & Piezoelectric patch (PI Instruments (2014)) & Base beam \\
\hline Material & PIC 155 & Aluminium \\
Density, $\rho\left(\mathrm{kg} \mathrm{m}^{-3}\right)$ & 7800 & 2700 \\
Young's modulus, $E(\mathrm{GPa})$ & 66.7 (Short circuit) & 69.0 \\
Shear modulus, $G(\mathrm{GPa})$ & 24.1 (Short circuit) & 25.9 \\
Shear coefficient, $\kappa$ & 0.85 & 0.85 \\
Relative permittivity at constant stress, $\frac{\epsilon_{33}^{T}}{\epsilon_{0}}$ & 2400 & N/A \\
Piezoelectric voltage coefficient, $d_{31}\left(\times 10^{-12} \mathrm{C} \mathrm{N}^{-1}\right)$ & -210 & N/A \\
\hline
\end{tabular}

Table 2. Geometries of piezoelectric patch and base beam.

\begin{tabular}{lcc}
\hline Dimension & Piezoelectric patch & Base beam \\
\hline Height, $h(\mathrm{~mm})$ & 0.5 & 3 \\
Width, $b(\mathrm{~mm})$ & 20 & 20 \\
Length, $L(\mathrm{~mm})$ & 20 & 20 (Portion without piezoelectric patch) \\
\hline
\end{tabular}

in the appendix.

$$
\begin{aligned}
& \mathbf{D}=\left[\begin{array}{ccc}
i \omega \mathbf{Z}_{N} & \mathbf{0} & \mathbf{P}_{N} \\
\mathbf{0} & i \omega \mathbf{Z}_{F} & \mathbf{P}_{F} \\
-\mathbf{P}_{N}^{T} & \mathbf{P}_{F}^{T} & \mathbf{C}
\end{array}\right] \\
& \mathbf{F}_{N}=\left[\begin{array}{ll}
-N(0) & N(l)
\end{array}\right]^{T} \\
& \mathbf{F}_{F}=\left[\begin{array}{llll}
-M(0) & -R(0) & M(l) & R(l)
\end{array}\right]^{T} \\
& \mathbf{I}=\left[\begin{array}{ll}
I^{t} & I^{b}
\end{array}\right]^{T} \\
& \mathbf{U}_{N}=[u(0) \quad u(l)]^{T} \\
& \mathbf{U}_{F}=\left[\begin{array}{llll}
\theta(0) & W(0) & \theta(l) & W(l)
\end{array}\right]^{T} \\
& \mathbf{V}=\left[\begin{array}{ll}
V^{t} & V^{b}
\end{array}\right]^{T}
\end{aligned}
$$

where $\mathbf{0}$ is a matrix of zeros, the superscript $T$ denotes transpose of a matrix, $N$ are the axial forces, $l$ is the length of the component, $M$ are the bending moments, $R$ are the transverse forces, $I$ are the currents, the superscripts $t$ and $b$ refers to the top and bottom patch respectively, $u$ are the axial displacements, $\theta$ are the angles of rotation due to bending, $W$ are the transverse displacements, and $V$ are the voltages.

For the portion of the beam without piezoelectric patches, the electrical components of the matrices in Eq. (3), which are $\mathbf{I}, \mathbf{V}, \mathbf{P}_{N}, \mathbf{P}_{F}$, and $\mathbf{C}$, were omitted. Furthermore, since a passive shunt was used and the piezoelectric components are not powered by an external voltage or current source, Eq. (3) can be simplified to Eq. (11), so that only the mechanical components are present as seen in Eq. (12).

$$
\begin{gathered}
V-I Z^{S U}=0 \\
{\left[\begin{array}{l}
\mathbf{F}_{N} \\
\mathbf{F}_{F}
\end{array}\right]=\mathbf{D}_{r}\left[\begin{array}{l}
\mathbf{U}_{N} \\
\mathbf{U}_{F}
\end{array}\right]}
\end{gathered}
$$

where

$$
\begin{aligned}
\mathbf{D}_{r}= & {\left[\begin{array}{cc}
i \omega \mathbf{Z}_{N} & \mathbf{0} \\
\mathbf{0} & i \omega \mathbf{Z}_{N}
\end{array}\right]-} \\
& {\left[\begin{array}{ll}
\mathbf{P}_{N} & \mathbf{0} \\
\mathbf{P}_{F} & \mathbf{0}
\end{array}\right]\left(\left[\begin{array}{ll}
\mathbf{C} & -\mathbf{1} \\
\mathbf{0} & Z^{S U}
\end{array}\right]^{-1}\left[\begin{array}{cc}
-\mathbf{P}_{N}^{T} & \mathbf{P}_{F}^{T} \\
\mathbf{0} & \mathbf{1}
\end{array}\right]\right) }
\end{aligned}
$$

where $\mathbf{1}$ is the identity matrix.

\section{Transfer matrix method to calculate band structure and attenuation constant}

In this study, the Floquet-Bloch theorem (Brillouin (1946)), described by Eq. (14), was used to determine the wave propagation characteristics of the beam with periodic shunted piezoelectric patches.

$$
U(x+L)=\lambda U(x)=e^{i k L} U(x)
$$

The term $k$ in Eq. (14) is known as the wave vector and it can be a complex value with imaginary, $\delta$, and real, $\mu$, parts, which are commonly known as the attenuation and propagation constants respectively. The propagation constant, $\mu$, describes the phase shift, while the attenuation constant, $\delta$, describes the amount of attenuation that the elastic wave experiences as it passes through the unit cell.

The transfer matrix method described by Eq. (15) was used to find the wave vectors in this study mainly because it is capable of determining the complex wave vectors. This capability is essential as the shunted piezoelectric patches will provide intrinsic damping, which will lead to the wave vectors being complex. Additionally, the vibration attenuation performance of the piezoelectric patches are to be evaluated using the attenuation constants. Eq. (15) is an eigenvalue problem that can be easily solved. Furthermore, since only the bending waves are of interest, the axial components in Eq. (3) and Eq. (12) were ignored.

$$
\left[\begin{array}{c}
\mathbf{U}_{R} \\
-\mathbf{F}_{R}
\end{array}\right]=\mathbf{T}\left[\begin{array}{l}
\mathbf{U}_{L} \\
\mathbf{F}_{L}
\end{array}\right]=\lambda\left[\begin{array}{l}
\mathbf{U}_{L} \\
\mathbf{F}_{L}
\end{array}\right]
$$

where $\mathbf{T}$ is the transfer matrix, $\mathbf{U}$ are the displacement matrices, $\mathbf{F}$ are the force matrices and the subscripts $L$ and $R$ denote the left end and the right end of the beam.

In this study, the portions of the beam with and without the piezoelectric patches were treated as two separate beams, each having its own dynamic stiffness, in which continuity and dynamic equilibrium are enforced at the interface. An effective dynamic stiffness, $\tilde{\mathbf{D}}$, for the single unit cell can then be found after simplifying and rearranging the matrix into the form shown in Eq. (16). 


$$
\left[\begin{array}{c}
\mathbf{F}_{L} \\
\mathbf{0} \\
\mathbf{0} \\
\mathbf{F}_{R}
\end{array}\right]=\tilde{\mathbf{D}}\left[\begin{array}{c}
\mathbf{U}_{L} \\
\mathbf{U}_{I} \\
\mathbf{F}_{I} \\
\mathbf{U}_{R}
\end{array}\right]
$$

where the subscript $I$ denotes the internal nodes.

Grouping the interface displacements and forces as a single matrix, Eq. (16) can be written as Eq. (17).

$$
\begin{aligned}
{\left[\begin{array}{c}
\mathbf{F}_{L} \\
\mathbf{0} \\
\mathbf{F}_{R}
\end{array}\right] } & =\tilde{\mathbf{D}}\left[\begin{array}{c}
\mathbf{U}_{L} \\
\mathbf{U}_{,} \mathbf{F}_{I} \\
\mathbf{U}_{R}
\end{array}\right] \\
& =\left[\begin{array}{ccc}
\tilde{\mathbf{D}}_{L, L} & \tilde{\mathbf{D}}_{L, I} & \tilde{\mathbf{D}}_{L, R} \\
\tilde{\mathbf{D}}_{I, L} & \tilde{\mathbf{D}}_{I, I} & \tilde{\mathbf{D}}_{I, R} \\
\tilde{\mathbf{D}}_{R, L} & \tilde{\mathbf{D}}_{R, I} & \tilde{\mathbf{D}}_{R, R}
\end{array}\right]\left[\begin{array}{c}
\mathbf{U}_{L} \\
\mathbf{U}, \mathbf{F}_{I} \\
\mathbf{U}_{R}
\end{array}\right]
\end{aligned}
$$

Using the steps taken by Spadoni et al. (2009), the transfer matrix, T, in Eq. (15) can be found as follows.

$$
\mathbf{T}=\left[\begin{array}{cc}
-\boldsymbol{\alpha}_{L, R}^{-1} \boldsymbol{\alpha}_{L, L} & \boldsymbol{\alpha}_{L, R}^{-1} \\
\boldsymbol{\alpha}_{R, R}\left(\boldsymbol{\alpha}_{L, R}^{-1} \boldsymbol{\alpha}_{L, L}\right)-\boldsymbol{\alpha}_{R, L} & -\boldsymbol{\alpha}_{R, R} \boldsymbol{\alpha}_{L, R}^{-1}
\end{array}\right]
$$

where

$$
\begin{aligned}
\boldsymbol{\alpha}_{L, L} & =\tilde{\mathbf{D}}_{L, L}-\tilde{\mathbf{D}}_{L, I}\left(\tilde{\mathbf{D}}_{I, I}^{-1} \tilde{\mathbf{D}}_{I, L}\right) \\
\boldsymbol{\alpha}_{L, R} & =\tilde{\mathbf{D}}_{L, R}-\tilde{\mathbf{D}}_{L, I}\left(\tilde{\mathbf{D}}_{I, I}^{-1} \tilde{\mathbf{D}}_{I, R}\right) \\
\boldsymbol{\alpha}_{R, L} & =\tilde{\mathbf{D}}_{R, L}-\tilde{\mathbf{D}}_{R, I}\left(\tilde{\mathbf{D}}_{I, I}^{-1} \tilde{\mathbf{D}}_{I, L}\right) \\
\boldsymbol{\alpha}_{R, R} & =\tilde{\mathbf{D}}_{R, R}-\tilde{\mathbf{D}}_{R, I}\left(\tilde{\mathbf{D}}_{I, I}^{-1} \tilde{\mathbf{D}}_{I, R}\right)
\end{aligned}
$$

A Matlab script was written to perform the required calculations to find the wave vectors, $k$, from the equations stated.

\section{Map calculations}

A common criterion used to quantify the effectiveness of wave attenuation within a phononic crystal or elastic metamaterial is the attenuation constant or the imaginary part of the wave vector, $\delta$. As there are 4 waves ( 2 incident and 2 reflected waves) that are present in the transverse bending of a beam, there will be two sets of wave vectors and hence two sets of attenuation constants, $\delta$, that characterises the waves. Here, the attenuation constant with the lowest magnitude will be used to quantify the wave attenuation properties because the waves with large attenuation attenuate more rapidly.

The attenuation constants, $\delta$, for the beam configuration described previously was evaluated for a range of frequencies from $1 \mathrm{~Hz}$ to $15000 \mathrm{~Hz}$, and shunting impedance defined in Eq. (11), with magnitudes, $a$, from 1 to $5000 \mathrm{~V} \mathrm{~A}^{-1}$ and phase angle, $\phi$, from $-\pi$ to $\pi$ rad. This enables the attenuation constants for all possible shunting circuits to be determined and the best possible shunt configuration can be identified.

In order to conserve computing resources and time, these attenuation constants were first evaluated at larger intervals for the phase angle and magnitude, which are $\frac{\pi}{10} \operatorname{rad}$ and $10 \mathrm{~V} \mathrm{~A}^{-1}$. It is important to point out that despite the large intervals taken for the magnitude and phase angle, the number of points that were evaluated is still very large $\left(21 \times 500 \times 15000=1.575 \times 10^{8}\right)$, where the attenuation constants were evaluated at every $1 \mathrm{~Hz}$ frequency interval. The attenuation constants in regions that have interesting features were then evaluated at smaller intervals of magnitude and phase angle.

\section{Results for attenuation constant map}

\section{Volume plot of attenuation constant}

A volume plot mapping the attenuation constant over a range of frequencies, magnitudes and phase angle of the shunt impedance is shown in Fig. 3. Due to the large intervals in $a$ and $\phi$ used in finding the attenuation constant, the plot in Fig. 3 was generated by first smoothing the 3D data using the smooth 3 function, then plotting the data using the isosurface function in Matlab (Mathworks (2014)).

In Fig. 3, each surface corresponds to a region with a constant attenuation constant, and the colour of the surfaces describes the magnitude of the attenuation constant for the isosurface. One observation that can be made from Fig. 3 is that there is a region from 4135 to $4375 \mathrm{~Hz}$ that has attenuation constants of above 0.025 regardless of the impedance of the shunt. The band gap produced at these frequencies is a Bragg gap, which is a result of the discontinuity in wave speed along the beam caused by the addition of the piezoelectric patch.

Another interesting observation in Fig. 3 is that there is a region centred around $\phi=\frac{\pi}{2}$ rad with high attenuation constant above 0.2 , suggesting very effective wave attenuation. This region will be referred to as the high attenuation zone herein. Additionally, the frequencies of the high attenuation zone appear to be decreasing exponentially with increasing magnitude of shunt impedance. This trend observed in Fig. 3 can be used to devise a strategy for the design of passive shunts to create effective band gaps. As seen in Fig. 3, the ideal passive shunt should have an impedance (both magnitude and phase angle) that follows the dark red region over the range of frequencies.

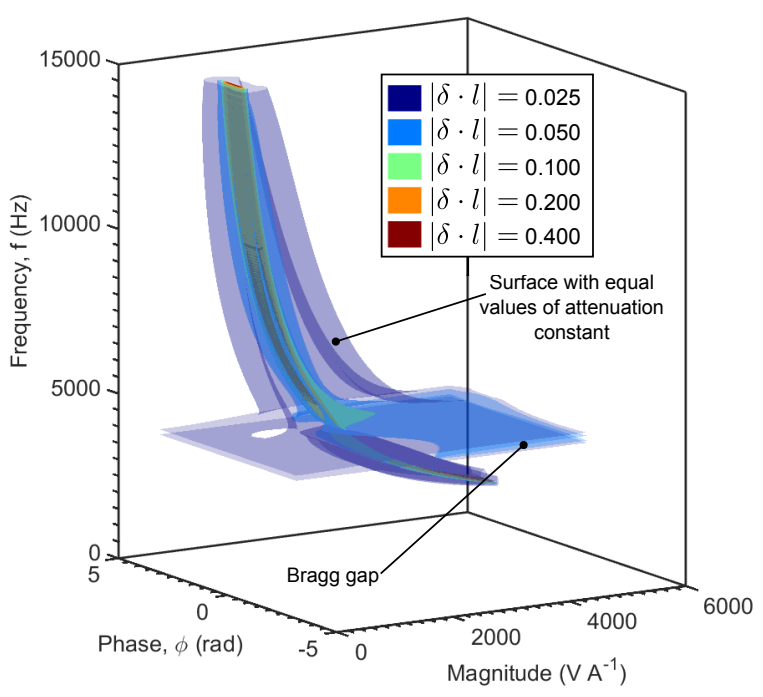

Figure 3. Isosurface plot of attenuation constant for different values of $a$ and $\phi$ over a range of frequencies. 
In order to investigate the trends further, a contour plot of the attenuation constants at a constant frequency, selected to be $6000 \mathrm{~Hz}$, is plotted in Fig. 4 to deduce the optimal phase angle for the shunt. As seen in Fig. 4 (a), the attenuation constant is maximum at a phase angle of $\phi=\frac{\pi}{2} \mathrm{rad}$. Since large intervals of $\phi$ were used in the calculations for Fig. 4 (a), the analysis was repeated with smaller intervals of 0.01 rad around $\phi=\frac{\pi}{2}$ rad in order to get a detailed representation of the attenuation constant. The contour plot with smaller intervals is shown in Fig. 4 (b) and the maximum attenuation constant still occurs at a phase angle of $\phi=\frac{\pi}{2} \mathrm{rad}$. The phase angle of $\phi=\frac{\pi}{2}$ rad suggests that the impedance of the optimal shunt is purely imaginary and has a positive value as described by Eq. (23).

$$
Z_{\text {Optimum }}=a(\omega) \cdot j
$$

where $a$ is a positive value denoting the magnitude of the impedance.

Additionally, as seen in Fig. 4 (b), the attenuation constant is very sensitive to both the magnitude and phase angle of the shunt impedance as the it decays rapidly from the optimal value. After establishing that the optimal shunt will have a phase angle of $\phi=\frac{\pi}{2}$ rad, a contour plot of the attenuation constant at $\phi=\frac{\pi}{2}$ rad, as shown in Fig. 5, was investigated. This is to determine the trend of the attenuation constant with the magnitude of the shunt impedance. As seen in Fig. 5, the frequencies of the high attenuation zone appear to be decreasing exponentially with the magnitude of the shunt impedance described by Eq. (24).

$$
f=\frac{1}{2 \pi B \cdot a^{n}}
$$

where $a$ is a positive value denoting the magnitude of the impedance and $B$ and $n$ are constants.

Since the phase angle is $\phi=\frac{\pi}{2} \mathrm{rad}$, the optimal shunt is likely to follow the equation described by Eq. (25).

$$
Z=\frac{j}{2 \pi \tilde{B}} f^{-n}
$$

where $\tilde{B}$ is a constant.

If the value of $n$ is unity in Eq. (25), this becomes the equation for the impedance of a negative capacitance circuit, shown in Eq. (26), which is similar to the circuits studied in (Chen et al. (2011); Tateo et al. (2014); Casadei et al. (2012)), but without any resistors in the circuit,

$$
Z=\frac{1}{2 j \pi f\left(-C_{p}\right)}
$$

where $C_{p}$ is the magnitude of the negative capacitance shunt.

The dashed line in Fig. 5 denotes the impedance of a negative capacitance shunt with the capacitance of -15.5 $\mathrm{nF}$, and it overlaps with the high attenuation zones. This suggest that negative capacitance shunts are highly suitable candidates to attenuate waves for periodic piezoelectric shunt configurations. This result is consistent with Eq. (1) although a different model for the piezoelectric patches was used. Furthermore, based on the current analysis and Fig. 3 to Fig. 5, the attenuation constants for the beams were found to be sensitive to the shunt impedance and the attenuation constant will reduce significantly when the impedance of the shunt deviates from the optimal value. Therefore, for a beam with periodic shunted piezoelectric patches, the ideal strategy is to match the impedance of the shunts to the values in the high attenuation zones across a large range of frequencies. Based on the results from Fig. 5, the optimal type of shunt will be that of a negative capacitance. In order to demonstrate this, the band structure of a periodic beam with piezoelectric patches using an $\mathrm{R}-\mathrm{L}$ shunt and a negative capacitance shunt will be compared.

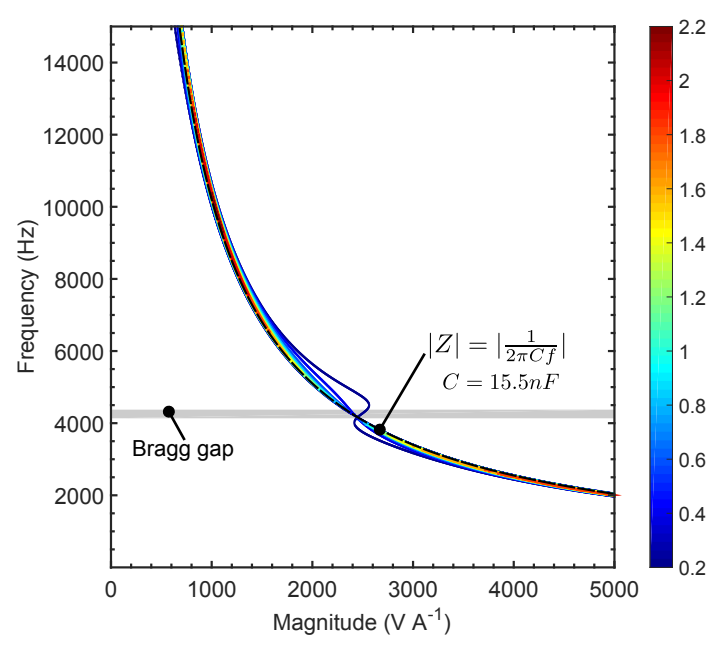

Figure 5. Contour plot of attenuation constant with varying values of $a$ over a range of frequencies at $\phi=\frac{\pi}{2}$.

\section{Comparison between R-L shunt and negative capacitance shunts}

According to the findings in the previous section, the shunting circuit should have impedance with a phase angle of $\frac{\pi}{2} \mathrm{rad}$ and a magnitude within the high attenuation zone shown in Fig. 5 to attenuate elastic waves with band gaps. In order to demonstrate that this is the case irrespective of the type of shunting circuit, the band structure and the transmissibilities of a finite periodic beam with different circuits will be investigated: a resistive-inductive (R-L) circuit, a negative capacitance shunt and a single resistor. These circuits have been considered in previous studies to generate band gaps with periodic piezoelectric patches.

The resistor for the R-L circuit was set to have a low resistance of $0.1 \mathrm{~m} \Omega$, and the inductance was set to be 23.28 $\mathrm{mH}$, to give a resonant frequency of $8000 \mathrm{~Hz}$ for the shunting circuit according to Eq. (27) (Chen et al. (2013b)). For the negative capacitance circuit, a low resistance of $0.1 \mathrm{~m} \Omega$ was set and the capacitance was set to be $-15.5 \mathrm{nF}$ to give the impedance that overlaps with the high attenuation region in Fig. 5. Lastly, the resistor only circuit was set to have a resistance of $1 \Omega$.

$$
f_{n}=\frac{1}{2 \pi \sqrt{L C_{p}}}
$$

where $f_{n}$ is the natural frequency of the shunt and piezoelectric patch circuit and $L$ is the inductance.

Since the R-L and negative capacitance circuits have negligible resistance, the phase angle of the impedance for 
(a)

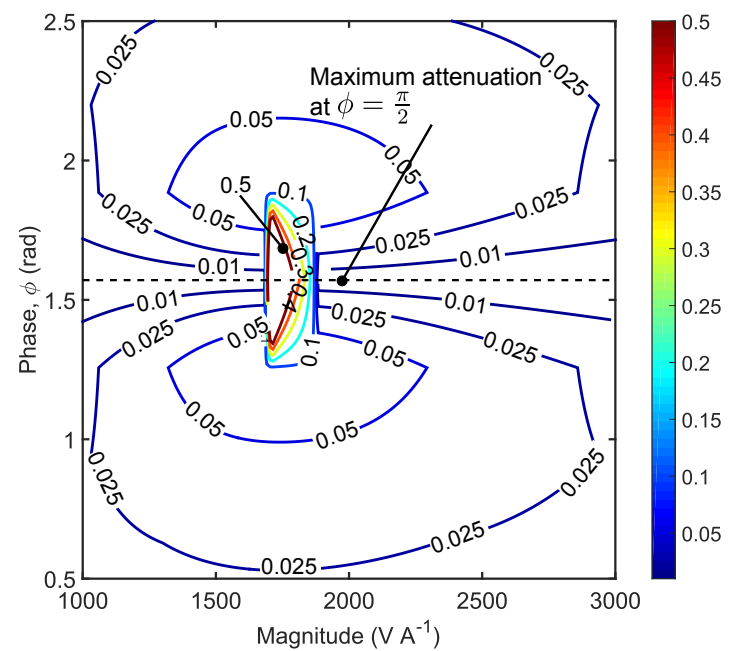

(b)

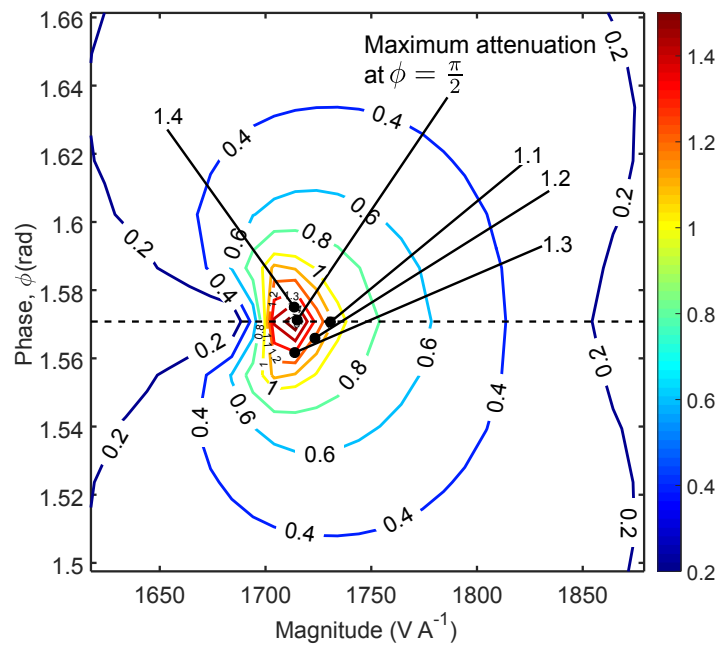

Figure 4. Contour plot of attenuation constant with varying values of $a$ and $\phi$ at $6000 \mathrm{~Hz}$. (a) Results from coarse sweep (b) Results from fine sweep.

the shunting circuits will be close to $\frac{\pi}{2}$ rad, while the phase angle of the impedance of the resistive shunt will always be 0 rad. Since the phase angle of the resistive shunt is far from the optimal value of $\frac{\pi}{2}$ rad, it will not produce any band gaps with the exception of the Bragg gap shown in Fig. 3. The band structure of the resistive circuit plotted in Fig. 6 shows that this is the case, where there is only a small band gap at 4151 to $4363 \mathrm{~Hz}$.

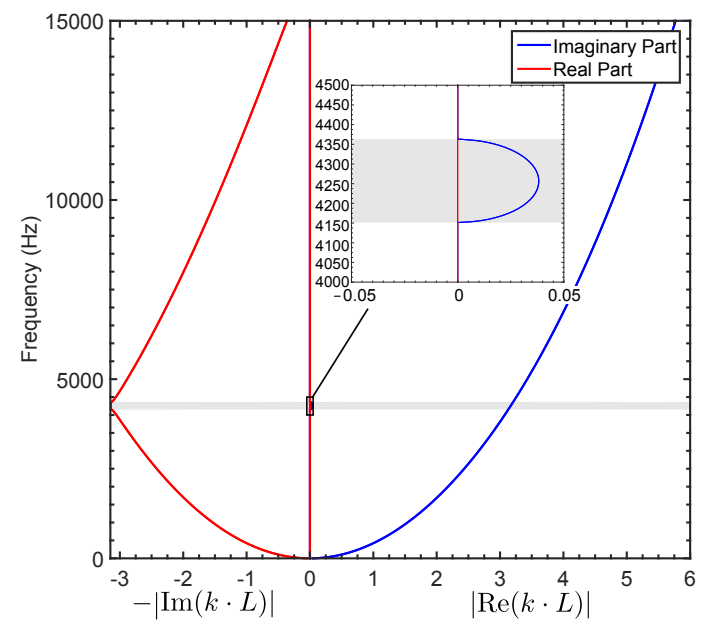

Figure 6. Band Structure of beam with $1 \Omega$ resistor.

The magnitudes of the impedance for the R-L and negative capacitance shunts are plotted along with the contours of the attenuation constants in Fig. 7. As seen in Fig. 7, the magnitude of the impedance of the negative capacitance circuit is within the high attenuation zone at frequencies above the Bragg gap and the impedance is on the boundary of the high attenuation zone at frequencies below that. Based on this result, the negative impedance circuit is expected to produce band gaps across the entire frequency range, which is the case as seen in the band structure shown in Fig. 8, where the imaginary part of the wave vectors above 4151
$\mathrm{Hz}$ is large. It is important to note that the imaginary part of the wave vectors at frequencies below $4151 \mathrm{~Hz}$ is very low but not zero, indicating that there is effectively no band gap at the lower frequencies. The negligible values of the imaginary part of the wave vectors at lower frequencies is a result of the impedance being close to the boundary of the high attenuation zone and as discussed in the previous section, the attenuation constants are very sensitive to the impedance and decays rapidly when the impedance deviates from the optimal value which is the case at lower frequencies.

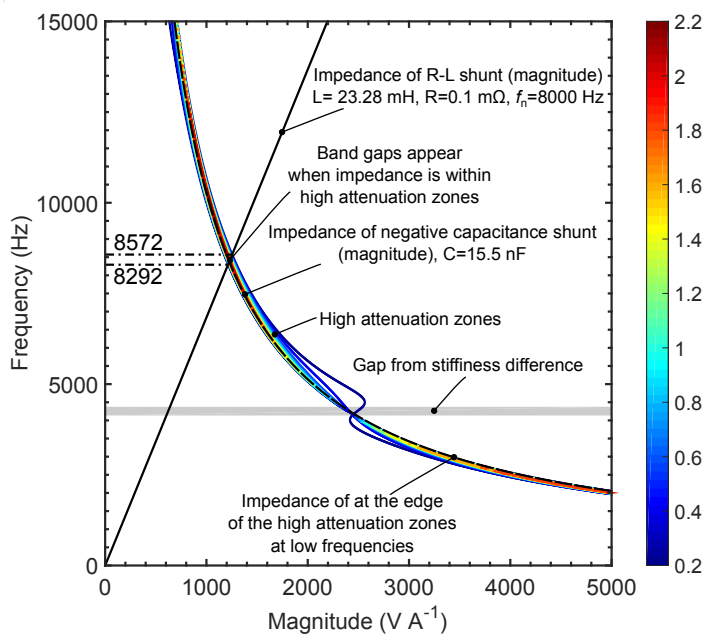

Figure 7. Contour plot of attenuation constants with varying values of $a$ over a range of frequencies at $\phi=\frac{\pi}{2}$ with different circuit configurations.

Furthermore, according to Fig. 7, the magnitude of the impedance of the R-L circuit varies linearly with excitation frequency. The impedance also overlaps with the high attenuation zones at frequencies in between 8292 and 8572 $\mathrm{Hz}$, which is around the tuned resonant frequency of 8000 Hz. In short, the impedance of the R-L circuit will overlap with the high attenuation zone around the tuned frequency. 


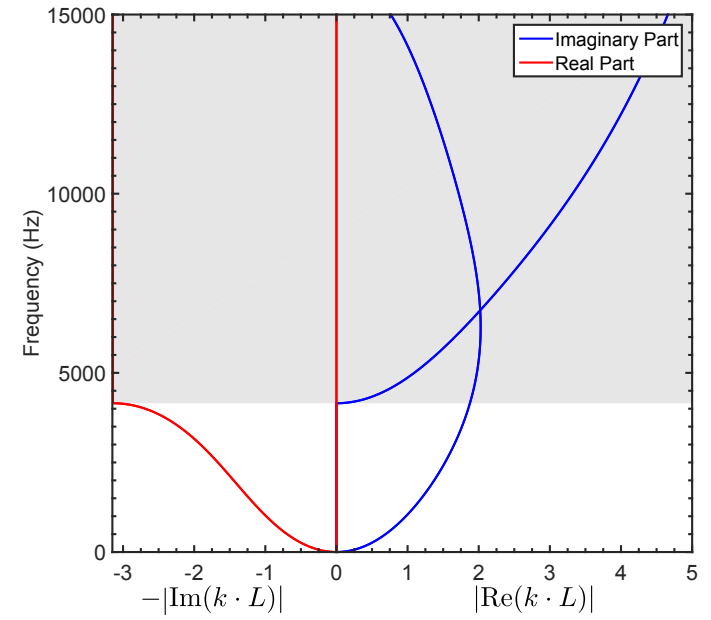

Figure 8. Band structure of beam with negative capacitance shunt.

Therefore, a band gap at this frequency range is expected and this is confirmed by inspecting the band structure of the periodic beam with the R-L shunt in Fig. 9. As seen in Fig. 9 there is a band gap in between frequencies of 8292 and 8572 $\mathrm{Hz}$ and a smaller Bragg gap at 4151 to $4319 \mathrm{~Hz}$.

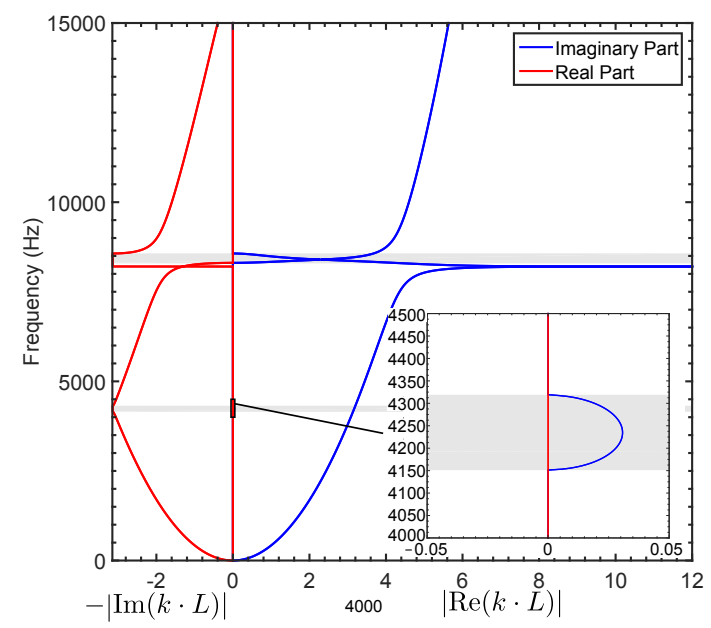

Figure 9. Band Structure of beam with R-L shunt.

The transmissibility curve defined as the magnitude of the ratio of the transverse displacements at the last node to that of the first node, for a finite periodic beam with 10 unit cells is shown in Fig. 10. The values calculated in Fig. 10 is for a free-free beam with a transverse force applied at one end of the beam. As seen in Fig. 10, there are no significant attenuation observed at frequencies where the imaginary wave vectors have very low values for all three circuits, for example the transmissibilities are still high at 4151 to $4363 \mathrm{~Hz}$ although there is a Bragg gap present. However, the transmissibilites are very low for regions where the imaginary wave vectors are large, for example at frequencies above $4151 \mathrm{~Hz}$ for the negative capacitance circuit and at frequencies in between 8292 to $8572 \mathrm{~Hz}$ for the R-L circuit. This result demonstrates the effectiveness of utilising the band gaps produced with the periodic shunted piezoelectric patches to attenuate elastic waves and vibrations.

The key finding from the analysis performed in this section is that effective band gaps will only be present when the phase angle of the shunt impedance is close to $\frac{\pi}{2} \mathrm{rad}$ and the magnitude must overlap with a region of high attenuation constants. Since the only region with high attenuation constant follows a trend where the magnitude decreases exponentially with the frequency, the only method to produce a band gap is to have the shunt impedance be in this region. This applies to all types of shunting circuits, including the R-L circuit that only produces the band gap when its impedance is within the attenuation zone. Therefore, in order to produce band gaps using periodic shunted piezoelectric patches, one should always set the impedance of the shunt to be within the high attenuation zone, which can be estimated by the impedance of a negative capacitance circuit with the magnitude of capacitance being approximately the value of the piezoelectric patch. Furthermore, based on the trends of the impedance of the R-L and negative capacitance shunts, it can be deduced that the negative capacitance shunts are capable of producing large band gaps compared to the R-L shunts as observed in Fig. 7, where the R-L shunt only has a small region of overlap.

Lastly, an important point to note is that the analyses done in this study did not take into account other issues, such as control system instabilities (Beck (2012)), the power consumption of the shunts, the operational limitations of the control system and circuits, and other factors that may be present in the practical implementation of such systems. Hence, care must be taken and some of these factors must be considered before implementing the system in practice. Despite the simplifications made in these analyses, the findings in this study give an overview of the general trend of the attenuation constants across all ranges of passive shunts for beam structures with periodic piezoelectric patches and the shunt design strategy described earlier can serve as a general guide in selecting the optimal shunts.

\section{Summary and conclusion}

Piezoelectric patches arranged periodically across a structure can be used to generate band gaps that are capable of attenuating vibrations when an appropriate shunting circuit is used. Since only two parameters, which are the phase angle, $\phi$, and magnitude, $a$, of the impedance, are required to characterise the entire shunt, the optimal shunt can be found by studying the attenuation constants across a range of frequencies for a beam with periodic piezoelectric patches across an entire range of $\phi$ and $a$.

Based on the volumetric plot showing the attenuation constants for different values of the excitation frequencies, phase angles and magnitudes of the shunt impedance, the optimal impedance was found to have a phase angle that is equal to $\frac{\pi}{2} \mathrm{rad}$ and has a magnitude that decreases exponentially with frequency. The optimal impedance was found to be similar to a negative capacitance shunt with a magnitude of capacitance that is approximately the capacitance of the piezoelectric patch. Additionally, a Bragg gap that is independent of the shunting circuit was found in a frequency range of 4151 to $4363 \mathrm{~Hz}$. Since the attenuation 
constant is very sensitive to the changes in impedance and reduces significantly when the impedance of the shunt deviates from the optimal value, a negative capacitance shunt will give the largest band gap as it is similar to the trend of the optimal impedance.

The band structures and impedances of several types of circuits, which are the R-L shunt, negative impedance shunt, and resistive shunt, have been studied. The resistive shunt was found to not produce any significant band gap as the phase angle of the resistor will always be constant at $0 \mathrm{rad}$. Conversely, the R-L and negative capacitance circuits have phase angles of $\frac{\pi}{2} \mathrm{rad}$ as they have negligible resistance. Therefore, these circuits are able to produce band gaps over a range of frequencies.

The negative capacitance shunt was found to produce a large band gap at frequencies above $4151 \mathrm{~Hz}$ as the impedance of the shunt is near the optimal value, while at frequencies below $4151 \mathrm{~Hz}$ the impedance is at the boundary of the attenuation zone and the attenuation constant is negligible in this case. On the other hand, the R-L circuit was found to have impedances within the high attenuation zone around the tuned resonant frequency of $8000 \mathrm{~Hz}$. The band gaps for the R-L circuits only appear within the frequency range where the impedance of the shunt overlaps with the high attenuation zones. This result suggests that regardless of circuit, one should set the impedance of the circuit to the values within the high attenuation zones to attain the most effective elastic wave attenuation with periodic shunted piezoelectric patches. Despite not taking into account some practical limitations, such as control system instabilities, the power consumption of the shunts, etc., the general trends of the attenuation constant with respect to the shunt parameters have been found and a strategy for designing the shunt for periodic piezoelectric patches was devised.

\section{Funding}

The strong support from the Aviation Industry Corporation of China (AVIC), the First Aircraft Institute (FAI) and Beijing Aeronautical Manufacturing Technology Research Institute (BAMTRI) for this funded research is much appreciated. The research was performed at the AVIC Centre for Structural Design and Manufacture at Imperial College London.

\section{Appendix: Terms in the transfer matrix}

The terms in the dynamic stiffness matrix, D, in Eq. (3) are as follows (Ha (2001)).

$\mathbf{Z}_{U}$ is a $2 \times 2$ symmetrical matrix with the non-zero terms defined as follows.

$$
\begin{gathered}
Z_{U}^{(1,1)}=Z_{U}^{(2,2)}=A_{11} \frac{\lambda_{0} \cos \left(\lambda_{0} l\right)}{j \omega \sin \left(\lambda_{0} l\right)} \\
Z_{U}^{(1,2)}=-A_{11} \frac{\lambda_{0}}{j \omega \sin \left(\lambda_{0} l\right)} \\
\lambda_{0}=\omega \sqrt{\frac{\rho_{h}}{A_{11}}} \\
\rho_{h}=\rho_{t} h_{t} b_{t}+\rho_{m} h_{m} b_{m}+\rho_{b} h_{b} b_{b}
\end{gathered}
$$

where $\rho$ is the density, $h$ is the thickness, $b$ is the width and the subscripts $t, m$ and $b$ denote the top, middle and bottom sections of the component.

$$
A_{11}=E_{t}^{E} h_{t} b_{t}+E_{m} h_{m} b_{m}+E_{b}^{E} h_{b} b_{b}
$$

where $E$ is the Young's modulus and the superscript $E$ denotes that the values are evaluated in the short circuit configuration. 
$\mathbf{Z}_{F}$ is a $4 \times 4$ symmetrical matrix with the non-zero terms defined as follows.

$$
\begin{aligned}
& Z_{F}^{(1,1)}=Z_{F}^{(3,3)} \\
& =\left(\frac{1}{X}\right) D_{11} \lambda_{a} \lambda_{b}\left(\lambda_{a}^{2}+\lambda_{b}^{2}\right)\left(\lambda_{b} \lambda_{1}^{2} c_{a} s_{b}-\lambda_{a} \lambda_{2}^{2} c_{b} s_{a}\right) \\
& Z_{F}^{(1,2)}=-Z_{F}^{(3,4)} \\
& =\left(\frac{1}{X}\right) D_{11} \rho_{D}\left[\lambda_{a} \lambda_{b}\left(\lambda_{1}^{2}-\lambda_{2}^{2}\right)\left(c_{a} c_{b}-1\right)\right. \\
& \left.\quad-\left(\lambda_{2}^{2} \lambda_{a}^{2}+\lambda_{1}^{2} \lambda_{b}^{2}\right)\left(s_{a} s_{b}\right)\right]
\end{aligned}
$$

$$
Z_{F}^{(1,3)}=\left(\frac{1}{X}\right) D_{11} \lambda_{a} \lambda_{b}\left(\lambda_{a}^{2}+\lambda_{b}^{2}\right)\left(\lambda_{a} \lambda_{2}^{2} s_{a}-\lambda_{b} \lambda_{1}^{2} s_{b}\right)
$$

$$
\begin{aligned}
Z_{F}^{(1,4)} & =-Z_{F}^{(2,3)} \\
& =-\left(\frac{1}{X}\right) D_{11} \rho_{D} \lambda_{a} \lambda_{b}\left(\lambda_{a}^{2}+\lambda_{b}^{2}\right)\left(c_{a}-c_{b}\right)
\end{aligned}
$$

$$
\begin{aligned}
& Z_{F}^{(2,2)}=Z_{F}^{(4,4)} \\
= & \left(\frac{1}{X}\right) D_{11} \rho_{D}\left(\lambda_{a}^{2}+\lambda_{b}^{2}\right)\left(\lambda_{a} \lambda_{2}^{2} c_{a} s_{b}-\lambda_{b} \lambda_{1}^{2} c_{b} s_{a}\right) \\
& Z_{F}^{(2,4)}=\left(\frac{1}{X}\right) D_{11} \rho_{D}\left(\lambda_{a}^{2}+\lambda_{b}^{2}\right)\left(\lambda_{a} \lambda_{2}^{2} s_{b}+\lambda_{b} \lambda_{1}^{2} s_{a}\right)
\end{aligned}
$$

$$
X=j \omega\left[2 \rho_{D} \lambda_{a} \lambda_{b}\left(c_{a} c_{b}-1\right)+s_{a} s_{b}\left(\lambda_{1}^{4} \lambda_{b}^{2}-\lambda_{2}^{4} \lambda_{a}^{2}\right)\right]
$$$$
c_{a}=\cos \left(\lambda_{a} l\right)
$$

$$
s_{a}=\sin \left(\lambda_{a} l\right)
$$

$$
c_{b}=\cosh \left(\lambda_{b} l\right)
$$$$
s_{b}=\sinh \left(\lambda_{b} l\right)
$$$$
\lambda_{1}^{2}=\lambda_{a}^{2}-\rho_{S}
$$$$
\lambda_{2}^{2}=\lambda_{b}^{2}+\rho_{S}
$$

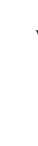

$$
\begin{gathered}
\rho_{S}=\frac{\rho_{h} \omega^{2}}{A_{55}} \\
\rho_{R}=\frac{\rho_{r} \omega^{2}}{D_{11}} \\
\rho_{D}=\frac{\rho_{h} \omega^{2}}{D_{11}} \\
\rho_{r}=\frac{\rho_{t} b_{t}}{3}\left[\left(\frac{h_{m}}{2}+h_{t}\right)^{3}-\left(\frac{h_{m}}{2}\right)^{3}\right]+\frac{\rho_{m} b_{m}}{12} h_{m}^{3}+ \\
\frac{\rho_{b} b_{b}}{3}\left[\left(\frac{h_{m}}{2}+h_{b}\right)^{3}-\left(\frac{h_{m}}{2}\right)^{3}\right]
\end{gathered}
$$

where $\rho$ are the densities.

$$
\begin{gathered}
D_{11}=\frac{E_{t}^{E} b_{t}}{3}\left[\left(\frac{h_{m}}{2}+h_{t}\right)^{3}-\left(\frac{h_{m}}{2}\right)^{3}\right]+\frac{E_{m} b_{m}}{12} h_{m}^{3}+ \\
\frac{E_{b}^{E} b_{b}}{3}\left[\left(\frac{h_{m}}{2}+h_{b}\right)^{3}-\left(\frac{h_{m}}{2}\right)^{3}\right] \\
A_{55}=\kappa\left(G_{t}^{E} b_{t} h_{t}+G_{m}^{E} b_{m} h_{m}+G_{b}^{E} b_{b} h_{b}\right)
\end{gathered}
$$

The electromechanical coupling matrices, $\mathbf{P}_{N}$ and $\mathbf{P}_{F}$, are defined as follows.

$$
\mathbf{P}_{N}=\left[\begin{array}{cc}
d_{31, t} E_{t}^{E} & d_{31, b} E_{b}^{E} \\
-d_{31, t} E_{t}^{E} & -d_{31, b} E_{b}^{E}
\end{array}\right]\left[\begin{array}{l}
b_{t} \\
b_{b}
\end{array}\right]
$$

where $d_{31}$ is the piezoelectric voltage coefficient.

$$
\mathbf{P}_{F}=\left[\begin{array}{cc}
-d_{31, t} E_{t}^{E} & -d_{31, b} E_{b}^{E} \\
0 & 0 \\
d_{31, t} E_{t}^{E} & d_{31, b} E_{b}^{E} \\
0 & 0
\end{array}\right]\left[\begin{array}{l}
b_{t} z_{t} \\
b_{t} z_{b}
\end{array}\right]
$$

The clamped admittance matrix, $\mathbf{C}$, is defined as follows.

$$
\mathbf{C}=i \omega l\left[\begin{array}{cc}
\epsilon_{33, t}^{T}-d_{31, t}^{2} E_{t}^{E} & 0 \\
0 & \epsilon_{33, b}^{T}-d_{31, b}^{2} E_{b}^{E}
\end{array}\right]\left[\begin{array}{c}
\frac{b_{t}}{h_{t}} \\
\frac{b_{b}}{h_{b}}
\end{array}\right]
$$

$$
\lambda_{b}=\sqrt{-\frac{1}{2}\left(\rho_{S}+\rho_{R}\right)+\sqrt{\frac{1}{4}\left(\rho_{S}-\rho_{R}\right)^{2}+\rho_{D}}}
$$

\section{References}

Airoldi L and Ruzzene M (2011) Design of tunable acoustic metamaterials through periodic arrays of resonant shunted piezos. New Journal of Physics 13(11): 113010.

Beck BS (2012) Negative capacitance shunting of piezoelectric patches for vibration control of continous systems .

Beck BS, Cunefare Ka, Collet M and Ruzzene M (2012) New Method of Negative Capacitance Shunt Tuning for Vibration Control 8341: 834106-834106-11.

Behrens S, Moheimani S and aJ Fleming (2003) Multiple mode current flowing passive piezoelectric shunt controller. Journal of Sound and Vibration 266(5): 929-942.

Brillouin L (1946) Wave Propagation In Periodic Structures. McGraw-Hill Book Company. 
Casadei F, Beck BS, Cunefare Ka and Ruzzene M (2012) Vibration control of plates through hybrid configurations of periodic piezoelectric shunts. Journal of Intelligent Material Systems and Structures 23(10): 1169-1177.

Chen S, Wang G, Wen J and Wen X (2013a) Wave propagation and attenuation in plates with periodic arrays of shunted piezopatches. Journal of Sound and Vibration 332(6): 1520-1532.

Chen SB, Wen JH, Wang G and Wen XS (2013b) Tunable band gaps in acoustic metamaterials with periodic arrays of resonant shunted piezos. Chinese Physics B 22(7): 074301.

Chen SB, Wen JH, Yu DL, Wang G and Wen XS (2011) Band gap control of phononic beam with negative capacitance piezoelectric shunt. Chinese Physics B 20(1): 014301.

Ha SK (2001) Analysis of the asymmetric triple-layered piezoelectric bimorph using equivalent circuit models. The Journal of the Acoustical Society of America 110(2): 856.

Hagood N and von Flotow A (1991) Damping of structural vibrations with piezoelectric materials and passive electrical networks. Journal of Sound and Vibration 146: 243-268.

Hussein MI, Leamy MJ and Ruzzene M (2014) Dynamics of Phononic Materials and Structures: Historical Origins, Recent Progress, and Future Outlook. Applied Mechanics Reviews 66(4): 040802.

Liu Z, Zhang X, Mao Y, Zhu Y, Yang Z, Chan C and Ping S (2000) Locally Resonant Sonic Materials. Science 289: 1734-1736.

Mathworks (2014) Matlab Documentaion.

PI Instruments (2014) Piezoelectric Ceramic Products: Fundamentals, characteristics and applications.

Spadoni A, Ruzzene M and Cunefare K (2009) Vibration and Wave Propagation Control of Plates with Periodic Arrays of Shunted Piezoelectric Patches. Journal of Intelligent Material Systems and Structures 20(8): 979-990.

Tateo F, Beck BS, Collet M, Ouisse M, Cunefare Ka and Ichchou MN (2014) Vibration control of plates through a periodic array of shunted piezoelectric patches with negative capacitance circuits 9057: 90572E.

Thorp O, Ruzzene M and Baz A (2001) Attenuation and localization of wave propagation in rods with periodic shunted piezoelectric patches. Smart Materials and Structures 979.

Wu Sy and McDonnell (1996) Piezoeleciric shunts with a parallel R-L circuit for structural damping and vibration control. Smart Structures and Materials 1996: Passive Damping and Isolation 27201259(Lc). 


\title{
Optimal shunt parameters for maximising wave attenuation with periodic piezoelectric patches
}

\author{
Journal Title \\ $\mathrm{XX}(\mathrm{X}): 1-10$ \\ (C) The Author(s) 2015 \\ Reprints and permission: \\ sagepub.co.uk/journalsPermissions.nav \\ DOI: 10.1177/ToBeAssigned \\ www.sagepub.com/ \\ SAGE_Logo-eps-converted-to.pdf
}

\author{
Lee Junyi ${ }^{1}$ and Daniel Stephen Balint ${ }^{1}$
}

\begin{abstract}
Elastic metamaterials, which have huge potential in wave guiding and attenuation applications, can be built from structures with periodic piezoelectric patch arrays. Passive shunts offer the benefits of simplicity and low cost. In this paper, the effects of the magnitude and phase angle of the shunt impedance on the attenuation constant of a beam with periodic piezoelectric patch arrays were studied in order to determine the optimal shunt that produces the widest and most effective band gaps. The attenuation constants were found to be large when the phase angle is $\frac{\pi}{2}$ rad and when the magnitude decreases exponentially with the excitation frequency. This corresponds to a negative capacitance circuit, which is the optimal shunt for such systems. The attenuation constant of the system reduces significantly when the impedance deviates from the optimal value suggesting other circuits are less effective. The impedance and band structure of resistive-inductive (R-L), negative capacitance and resistive shunts were investigated. As expected, the negative capacitance circuit produces a large band gap, while the $R$ - $L$ circuit only produces a band gap around its natural frequency. The transmissibilities of a finite system with these circuits demonstrated that vibration transmissions are low within the band gaps.
\end{abstract}

\section{Keywords}

Band gap, Bloch wave theory, band structure, phononic crystals, elastic metamaterials, periodic structures, piezoelectric patches, passive shunt

\section{Introduction}

Piezoelectric patches have been widely considered in vibration damping and control applications in structures as they have the ability to convert electrical energy to mechanical energy and vice versa (Hagood and von Flotow (1991)). This allows the implementation of a variety of control strategies to damp vibrations. The passive shunt configuration, in which the piezoelectric patch is connected to a passive circuit, is considered to be one of the simplest control system because it avoids the need of complex amplifiers and sensing equipment (Hagood and von Flotow (1991)) required in active control strategies. Several configurations of passive piezoelectric patches have been proposed, for example the resistive-inductive (R-L) circuit (Hagood and von Flotow (1991)) that acts as a dynamic vibration absorber reducing vibrations at the tuned frequencies. Later studies have introduced other circuits for shunted piezoelectric patches that makes use of different concepts, including multiple resonating circuits (Wu and McDonnell (1996); Behrens et al. (2003)) and negative capacitance shunts (Beck (2012)).

With increasing interest in elastic metamaterials, piezoelectric patches arranged in periodic arrays (Thorp et al. (2001); Spadoni et al. (2009); Airoldi and Ruzzene (2011); Chen et al. (2013a,b); Casadei et al. (2012)) have been studied in order to create elastic metamaterials with tunable band gaps, which is a desired property for this class of materials (Hussein et al. (2014)). Unlike conventional configurations for vibration attenuation, the periodic shunted piezoelectric patch arrangement creates band gaps, which are frequency ranges in which wave propagation cannot occur, to attenuate elastic waves without targeting a given mode of vibration (Spadoni et al. (2009)). This strategy results in broadband vibration attenuation as opposed to damping at specific frequencies typical of conventional systems. Two common circuits used to introduce band gaps are the R-L circuit (Spadoni et al. (2009); Airoldi and Ruzzene (2011); Chen et al. (2013a)) and the negative capacitance circuit (Tateo et al. (2014); Beck et al. (2012)). For the R-L circuit, the band gaps are created via the local resonant mechanism in elastic metamaterials proposed by (Liu et al. (2000)) with the periodic piezoelectric patches acting as the tuned resonators. Negative capacitance shunts on the other hand have been known to reduce vibrations over a large range of frequencies. This is done by tuning the capacitance of the shunt to values close to the capacitance of the shunt so that the effective stiffness of the patch defined by Eq. (1) (Hagood and von Flotow (1991)) is sufficiently large to prevent motion from passing through. Hence, by using a periodic piezoelectric patch with negative capacitance, large mechanical impedance mismatch can be achieved to create wide band-gaps for vibration attenuation.

${ }^{1}$ Imperial College London, UK

Corresponding author:

Lee Junyi, Imperial College London, Mechanical Engineering Department, South Kensington Campus, London SW7 2AZ.

Email: junyi.lee108@imperial.ac.uk 


$$
E_{p}^{S U}(\omega)=E_{p}^{D}\left(1-\frac{k_{31}^{2}}{1+i \omega C_{p}^{\varepsilon} Z^{S U}(\omega)}\right)
$$

where $E_{p}^{S U}$ is the effective Young's Modulus of the piezoelectric patch, $E_{p}^{D}$ is the Young's Modulus of the piezoelectric patch at constant electrical displacement, $\omega$ is the excitation frequency, $k_{31}$ is the electro-mechanical coupling coefficient, $C_{p}^{\varepsilon}$ is the capacitance and $Z^{S U}$ is the impedance of the shunt.

Since the shunt parameters, which are the inductance, $L$, capacitance, $C$, and resistance, $R$, will affect the vibration attenuation performance of the periodic arrays, many studies (Thorp et al. (2001); Spadoni et al. (2009); Chen et al. (2013a)) have analysed the effects of these parameters on the band gap width, location, and magnitude of the attenuation constants for plates or beams with periodic piezoelectric patches. However, in those studies, the shunting circuit was selected prior to the analysis of the effects of the parameters on the band gaps. This limits the analyses to the selected circuit while neglecting the possibility that other circuit configurations might have better performance. Regardless of the selected circuit, the electrical impedance of the passive shunt, $Z^{S U}$, can be expressed in terms of only the magnitude, $a$, and the phase angle, $\phi$, using Eq. (2).

$$
Z^{S U}=a \cdot e^{i \phi}
$$

Therefore, the wave attenuation characteristics of all possible passive shunts can be studied by analysing only $a$ and $\phi$. Hence, a map of the attenuation constant over a range of frequencies and shunt impedances will provide valuable information to the design of shunts for elastic wave attenuation using periodic shunted piezoelectric patches. In this study, the attenuation constants for a beam with periodic piezoelectric patches will be calculated over a range of magnitudes and phase angles of the shunt impedance. The map of the attenuation constants will then be analysed to devise design strategies for the passive shunts.

This paper is organised as follows. Firstly, the beam and piezoelectric parameters, the models for the beam and piezoelectric patches, and the method used to calculate the attenuation constants will be described. After that, a map, which is a volume visualisation of the attenuation constants with respect to excitation frequency, magnitude and phase angle of shunt impedances will be presented. Then, the trends observed in the map and a suitable design strategy for the passive shunt will be discussed. Additionally, the band structure of the R-L and negative capacitance shunts will be discussed with regards to the map, and the optimal shunt configuration will be found.

\section{Description of beam and models}

\section{Beam geometry}

An infinite beam with piezoelectric patches, as seen in Fig. 1, was studied. A beam was selected instead of a plate mainly because the elastic wave in a beam only travels in a single direction, which simplifies the study as only the attenuation constant in one dimension needs to be considered. Maps for plates that have additional dimensions can be found using the same analysis and similar trends are expected to be found for plates with similar configurations.

(a)

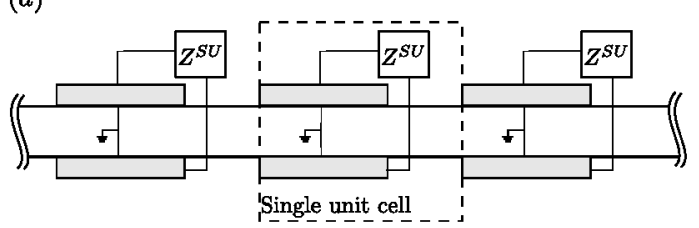

(b)

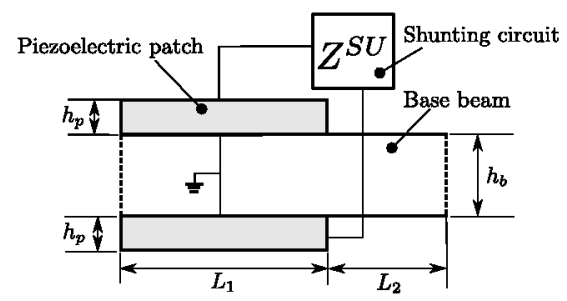

Figure 1. Infinite beam with periodic piezoelectric patch (a) A structure with infinitely repeating unit cells (b) A single unit cell.

The properties of the piezoelectric patch and beam used in this study are listed in Table 1 while the geometry of the unit cell is listed in Table 2. The shunting circuits for the top and bottom piezoelectric patch were set to be identical.

\section{Beam and piezoelectric model}

The model proposed by Ha (2001) was used to model the beam and piezoelectric patch in this study. This model was selected mainly because the Timoshenko beam model, which takes into account rotational inertia, results in more accurate predictions, especially at higher frequencies. Additionally, the model proposed by $\mathrm{Ha}$ (2001) allows the piezoelectric patch to be used as both sensor and actuator, which allows the same model to be used in future studies involving active control.

In the Ha (2001) model, the dynamic behaviour of the piezoelectric patch and the beam, using the sign convention shown in Fig. 2, is described by the transfer matrix in Eq. (3).

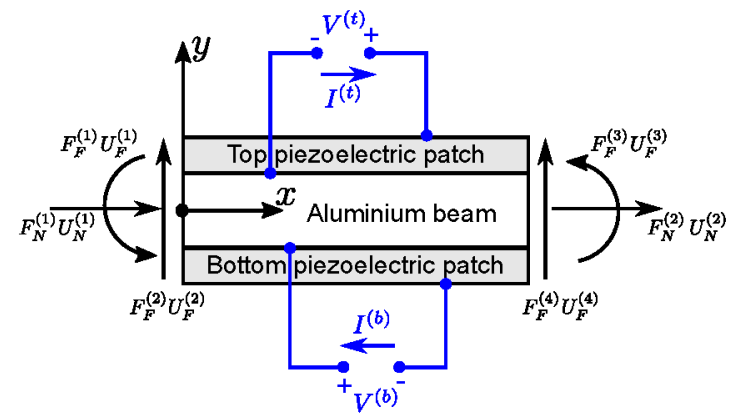

Figure 2. Piezoelectric patch and beam model.

$$
\left[\begin{array}{c}
\mathbf{F}_{N} \\
\mathbf{F}_{F} \\
\mathbf{I}
\end{array}\right]=\mathbf{D}\left[\begin{array}{c}
\mathbf{U}_{N} \\
\mathbf{U}_{F} \\
\mathbf{V}
\end{array}\right]
$$

where the terms are described by Eqs. (4) to (10); the terms within the dynamic stiffness matrix, D, in Eq. (4) are listed 
Table 1. Properties of piezoelectric patch and base beam.

\begin{tabular}{lcc}
\hline Property & Piezoelectric patch (PI Instruments (2014)) & Base beam \\
\hline Material & PIC 155 & Aluminium \\
Density, $\rho\left(\mathrm{kg} \mathrm{m}^{-3}\right)$ & 7800 & 2700 \\
Young's modulus, $E(\mathrm{GPa})$ & 66.7 (Short circuit) & 69.0 \\
Shear modulus, $G(\mathrm{GPa})$ & 24.1 (Short circuit) & 25.9 \\
Shear coefficient, $\kappa$ & 0.85 & 0.85 \\
Relative permittivity at constant stress, $\frac{\epsilon_{33}^{T}}{\epsilon_{0}}$ & 2400 & N/A \\
Piezoelectric voltage coefficient, $d_{31}\left(\times 10^{-12} \mathrm{C} \mathrm{N}^{-1}\right)$ & -210 & N/A \\
\hline
\end{tabular}

Table 2. Geometries of piezoelectric patch and base beam.

\begin{tabular}{lcc}
\hline Dimension & Piezoelectric patch & Base beam \\
\hline Height, $h(\mathrm{~mm})$ & 0.5 & 3 \\
Width, $b(\mathrm{~mm})$ & 20 & 20 \\
Length, $L(\mathrm{~mm})$ & 20 & 20 (Portion without piezoelectric patch) \\
\hline
\end{tabular}

in the appendix.

$$
\begin{aligned}
& \mathbf{D}=\left[\begin{array}{ccc}
i \omega \mathbf{Z}_{N} & \mathbf{0} & \mathbf{P}_{N} \\
\mathbf{0} & i \omega \mathbf{Z}_{F} & \mathbf{P}_{F} \\
-\mathbf{P}_{N}^{T} & \mathbf{P}_{F}^{T} & \mathbf{C}
\end{array}\right] \\
& \mathbf{F}_{N}=\left[\begin{array}{ll}
-N(0) & N(l)
\end{array}\right]^{T} \\
& \mathbf{F}_{F}=\left[\begin{array}{llll}
-M(0) & -R(0) & M(l) & R(l)
\end{array}\right]^{T} \\
& \mathbf{I}=\left[\begin{array}{ll}
I^{t} & I^{b}
\end{array}\right]^{T} \\
& \mathbf{U}_{N}=\left[\begin{array}{ll}
u(0) & u(l)
\end{array}\right]^{T} \\
& \mathbf{U}_{F}=\left[\begin{array}{llll}
\theta(0) & W(0) & \theta(l) & W(l)
\end{array}\right]^{T} \\
& \mathbf{V}=\left[\begin{array}{ll}
V^{t} & V^{b}
\end{array}\right]^{T}
\end{aligned}
$$

where $\mathbf{0}$ is a matrix of zeros, the superscript $T$ denotes transpose of a matrix, $N$ are the axial forces, $l$ is the length of the component, $M$ are the bending moments, $R$ are the transverse forces, $I$ are the currents, the superscripts $t$ and $b$ refers to the top and bottom patch respectively, $u$ are the axial displacements, $\theta$ are the angles of rotation due to bending, $W$ are the transverse displacements, and $V$ are the voltages.

For the portion of the beam without piezoelectric patches, the electrical components of the matrices in Eq. (3), which are $\mathbf{I}, \mathbf{V}, \mathbf{P}_{N}, \mathbf{P}_{F}$, and $\mathbf{C}$, were omitted. Furthermore, since a passive shunt was used and the piezoelectric components are not powered by an external voltage or current source, Eq. (3) can be simplified to Eq. (11), so that only the mechanical components are present as seen in Eq. (12).

$$
\begin{gathered}
V-I Z^{S U}=0 \\
{\left[\begin{array}{l}
\mathbf{F}_{N} \\
\mathbf{F}_{F}
\end{array}\right]=\mathbf{D}_{r}\left[\begin{array}{l}
\mathbf{U}_{N} \\
\mathbf{U}_{F}
\end{array}\right]}
\end{gathered}
$$

where

$$
\begin{aligned}
\mathbf{D}_{r}= & {\left[\begin{array}{cc}
i \omega \mathbf{Z}_{N} & \mathbf{0} \\
\mathbf{0} & i \omega \mathbf{Z}_{N}
\end{array}\right]-} \\
& {\left[\begin{array}{ll}
\mathbf{P}_{N} & \mathbf{0} \\
\mathbf{P}_{F} & \mathbf{0}
\end{array}\right]\left(\left[\begin{array}{cc}
\mathbf{C} & -\mathbf{1} \\
\mathbf{0} & Z^{S U} \mathbf{1}
\end{array}\right]^{-1}\left[\begin{array}{cc}
-\mathbf{P}_{N}^{T} & \mathbf{P}_{F}^{T} \\
\mathbf{0} & \mathbf{1}
\end{array}\right]\right) }
\end{aligned}
$$

where $\mathbf{1}$ is the identity matrix.

\section{Transfer matrix method to calculate band structure and attenuation constant}

In this study, the Floquet-Bloch theorem (Brillouin (1946)), described by Eq. (14), was used to determine the wave propagation characteristics of the beam with periodic shunted piezoelectric patches.

$$
U(x+L)=\lambda U(x)=e^{i k L} U(x)
$$

The term $k$ in Eq. (14) is known as the wave vector and it can be a complex value with imaginary, $\delta$, and real, $\mu$, parts, which are commonly known as the attenuation and propagation constants respectively. The propagation constant, $\mu$, describes the phase shift, while the attenuation constant, $\delta$, describes the amount of attenuation that the elastic wave experiences as it passes through the unit cell.

The transfer matrix method described by Eq. (15) was used to find the wave vectors in this study mainly because it is capable of determining the complex wave vectors. This capability is essential as the shunted piezoelectric patches will provide intrinsic damping, which will lead to the wave vectors being complex. Additionally, the vibration attenuation performance of the piezoelectric patches are to be evaluated using the attenuation constants. Eq. (15) is an eigenvalue problem that can be easily solved. Furthermore, since only the bending waves are of interest, the axial components in Eq. (3) and Eq. (12) were ignored.

$$
\left[\begin{array}{c}
\mathbf{U}_{R} \\
-\mathbf{F}_{R}
\end{array}\right]=\mathbf{T}\left[\begin{array}{l}
\mathbf{U}_{L} \\
\mathbf{F}_{L}
\end{array}\right]=\lambda\left[\begin{array}{l}
\mathbf{U}_{L} \\
\mathbf{F}_{L}
\end{array}\right]
$$

where $\mathbf{T}$ is the transfer matrix, $\mathbf{U}$ are the displacement matrices, $\mathbf{F}$ are the force matrices and the subscripts $L$ and $R$ denote the left end and the right end of the beam.

In this study, the portions of the beam with and without the piezoelectric patches were treated as two separate beams, each having its own dynamic stiffness, in which continuity and dynamic equilibrium are enforced at the interface. An effective dynamic stiffness, $\tilde{\mathbf{D}}$, for the single unit cell can then be found after simplifying and rearranging the matrix into the form shown in Eq. (16). 


$$
\left[\begin{array}{c}
\mathbf{F}_{L} \\
\mathbf{0} \\
\mathbf{0} \\
\mathbf{F}_{R}
\end{array}\right]=\tilde{\mathbf{D}}\left[\begin{array}{c}
\mathbf{U}_{L} \\
\mathbf{U}_{I} \\
\mathbf{F}_{I} \\
\mathbf{U}_{R}
\end{array}\right]
$$

where the subscript $I$ denotes the internal nodes.

Grouping the interface displacements and forces as a single matrix, Eq. (16) can be written as Eq. (17).

$$
\begin{aligned}
{\left[\begin{array}{c}
\mathbf{F}_{L} \\
\mathbf{0} \\
\mathbf{F}_{R}
\end{array}\right] } & =\tilde{\mathbf{D}}\left[\begin{array}{c}
\mathbf{U}_{L} \\
\mathbf{U}, \mathbf{F}_{I} \\
\mathbf{U}_{R}
\end{array}\right] \\
& =\left[\begin{array}{lll}
\tilde{\mathbf{D}}_{L, L} & \tilde{\mathbf{D}}_{L, I} & \tilde{\mathbf{D}}_{L, R} \\
\tilde{\mathbf{D}}_{I, L} & \tilde{\mathbf{D}}_{I, I} & \tilde{\mathbf{D}}_{I, R} \\
\tilde{\mathbf{D}}_{R, L} & \tilde{\mathbf{D}}_{R, I} & \tilde{\mathbf{D}}_{R, R}
\end{array}\right]\left[\begin{array}{c}
\mathbf{U}_{L} \\
\mathbf{U}, \mathbf{F}_{I} \\
\mathbf{U}_{R}
\end{array}\right]
\end{aligned}
$$

Using the steps taken by Spadoni et al. (2009), the transfer matrix, $\mathrm{T}$, in Eq. (15) can be found as follows.

$$
\mathbf{T}=\left[\begin{array}{cc}
-\boldsymbol{\alpha}_{L, R}^{-1} \boldsymbol{\alpha}_{L, L} & \boldsymbol{\alpha}_{L, R}^{-1} \\
\boldsymbol{\alpha}_{R, R}\left(\boldsymbol{\alpha}_{L, R}^{-1} \boldsymbol{\alpha}_{L, L}\right)-\boldsymbol{\alpha}_{R, L} & -\boldsymbol{\alpha}_{R, R} \boldsymbol{\alpha}_{L, R}^{-1}
\end{array}\right]
$$

where

$$
\begin{array}{r}
\boldsymbol{\alpha}_{L, L}=\tilde{\mathbf{D}}_{L, L}-\tilde{\mathbf{D}}_{L, I}\left(\tilde{\mathbf{D}}_{I, I}^{-1} \tilde{\mathbf{D}}_{I, L}\right) \\
\boldsymbol{\alpha}_{L, R}=\tilde{\mathbf{D}}_{L, R}-\tilde{\mathbf{D}}_{L, I}\left(\tilde{\mathbf{D}}_{I, I}^{-1} \tilde{\mathbf{D}}_{I, R}\right) \\
\boldsymbol{\alpha}_{R, L}=\tilde{\mathbf{D}}_{R, L}-\tilde{\mathbf{D}}_{R, I}\left(\tilde{\mathbf{D}}_{I, I}^{-1} \tilde{\mathbf{D}}_{I, L}\right) \\
\boldsymbol{\alpha}_{R, R}=\tilde{\mathbf{D}}_{R, R}-\tilde{\mathbf{D}}_{R, I}\left(\tilde{\mathbf{D}}_{I, I}^{-1} \tilde{\mathbf{D}}_{I, R}\right)
\end{array}
$$

A Matlab script was written to perform the required calculations to find the wave vectors, $k$, from the equations stated.

\section{Map calculations}

A common criterion used to quantify the effectiveness of wave attenuation within a phononic crystal or elastic metamaterial is the attenuation constant or the imaginary part of the wave vector, $\delta$. As there are 4 waves ( 2 incident and 2 reflected waves) that are present in the transverse bending of a beam, there will be two sets of wave vectors and hence two sets of attenuation constants, $\delta$, that characterises the waves. Here, the attenuation constant with the lowest magnitude will be used to quantify the wave attenuation properties because the waves with large attenuation attenuate more rapidly.

The attenuation constants, $\delta$, for the beam configuration described previously was evaluated for a range of frequencies from $1 \mathrm{~Hz}$ to $15000 \mathrm{~Hz}$, and shunting impedance defined in Eq. (11), with magnitudes, $a$, from 1 to $5000 \mathrm{~V} \mathrm{~A}^{-1}$ and phase angle, $\phi$, from $-\pi$ to $\pi$ rad. This enables the attenuation constants for all possible shunting circuits to be determined and the best possible shunt configuration can be identified.

In order to conserve computing resources and time, these attenuation constants were first evaluated at larger intervals for the phase angle and magnitude, which are $\frac{\pi}{10} \mathrm{rad}$ and $10 \mathrm{~V} \mathrm{~A}^{-1}$. It is important to point out that despite the large intervals taken for the magnitude and phase angle, the number of points that were evaluated is still very large $\left(21 \times 500 \times 15000=1.575 \times 10^{8}\right)$, where the attenuation constants were evaluated at every $1 \mathrm{~Hz}$ frequency interval. The attenuation constants in regions that have interesting features were then evaluated at smaller intervals of magnitude and phase angle.

\section{Results for attenuation constant map}

\section{Volume plot of attenuation constant}

A volume plot mapping the attenuation constant over a range of frequencies, magnitudes and phase angle of the shunt impedance is shown in Fig. 3. Due to the large intervals in $a$ and $\phi$ used in finding the attenuation constant, the plot in Fig. 3 was generated by first smoothing the 3D data using the smooth 3 function, then plotting the data using the isosurface function in Matlab (Mathworks (2014)).

In Fig. 3, each surface corresponds to a region with a constant attenuation constant, and the colour of the surfaces describes the magnitude of the attenuation constant for the isosurface. One observation that can be made from Fig. 3 is that there is a region from 4135 to $4375 \mathrm{~Hz}$ that has attenuation constants of above 0.025 regardless of the impedance of the shunt. The band gap produced at these frequencies is a Bragg gap, which is a result of the discontinuity in wave speed along the beam caused by the addition of the piezoelectric patch.

Another interesting observation in Fig. 3 is that there is a region centred around $\phi=\frac{\pi}{2}$ rad with high attenuation constant above 0.2 , suggesting very effective wave attenuation. This region will be referred to as the high attenuation zone herein. Additionally, the frequencies of the high attenuation zone appear to be decreasing exponentially with increasing magnitude of shunt impedance. This trend observed in Fig. 3 can be used to devise a strategy for the design of passive shunts to create effective band gaps. As seen in Fig. 3, the ideal passive shunt should have an impedance (both magnitude and phase angle) that follows the dark red region over the range of frequencies.

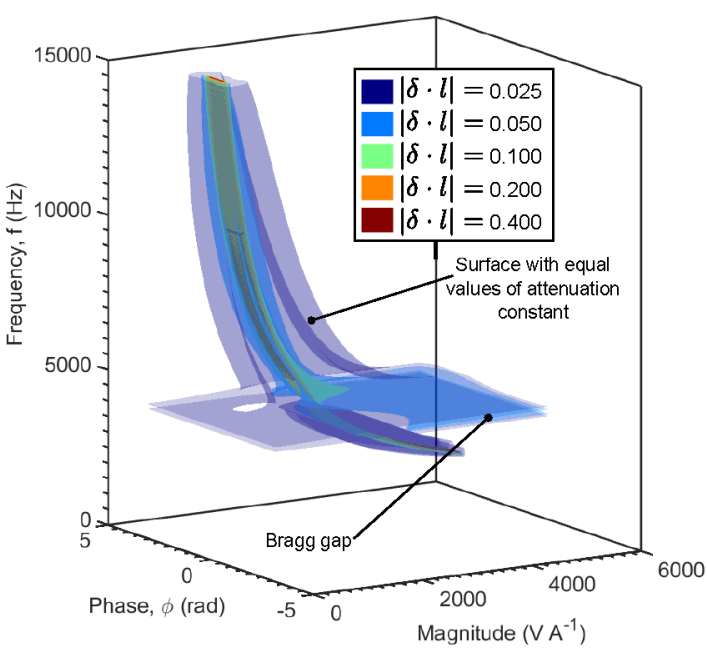

Figure 3. Isosurface plot of attenuation constant for different values of $a$ and $\phi$ over a range of frequencies. 
In order to investigate the trends further, a contour plot of the attenuation constants at a constant frequency, selected to be $6000 \mathrm{~Hz}$, is plotted in Fig. 4 to deduce the optimal phase angle for the shunt. As seen in Fig. 4 (a), the attenuation constant is maximum at a phase angle of $\phi=\frac{\pi}{2}$ rad. Since large intervals of $\phi$ were used in the calculations for Fig. 4 (a), the analysis was repeated with smaller intervals of 0.01 rad around $\phi=\frac{\pi}{2}$ rad in order to get a detailed representation of the attenuation constant. The contour plot with smaller intervals is shown in Fig. 4 (b) and the maximum attenuation constant still occurs at a phase angle of $\phi=\frac{\pi}{2} \mathrm{rad}$. The phase angle of $\phi=\frac{\pi}{2}$ rad suggests that the impedance of the optimal shunt is purely imaginary and has a positive value as described by Eq. (23).

$$
Z_{\text {Optimum }}=a(\omega) \cdot j
$$

where $a$ is a positive value denoting the magnitude of the impedance.

Additionally, as seen in Fig. 4 (b), the attenuation constant is very sensitive to both the magnitude and phase angle of the shunt impedance as the it decays rapidly from the optimal value. After establishing that the optimal shunt will have a phase angle of $\phi=\frac{\pi}{2} \mathrm{rad}$, a contour plot of the attenuation constant at $\phi=\frac{\pi}{2} \mathrm{rad}$, as shown in Fig. 5, was investigated. This is to determine the trend of the attenuation constant with the magnitude of the shunt impedance. As seen in Fig. 5, the frequencies of the high attenuation zone appear to be decreasing exponentially with the magnitude of the shunt impedance described by Eq. (24).

$$
f=\frac{1}{2 \pi B \cdot a^{n}}
$$

where $a$ is a positive value denoting the magnitude of the impedance and $B$ and $n$ are constants.

Since the phase angle is $\phi=\frac{\pi}{2} \mathrm{rad}$, the optimal shunt is likely to follow the equation described by Eq. (25).

$$
Z=\frac{j}{2 \pi \tilde{B}} f^{-n}
$$

where $\tilde{B}$ is a constant.

If the value of $n$ is unity in Eq. (25), this becomes the equation for the impedance of a negative capacitance circuit, shown in Eq. (26), which is similar to the circuits studied in (Chen et al. (2011); Tateo et al. (2014); Casadei et al. (2012)), but without any resistors in the circuit,

$$
Z=\frac{1}{2 j \pi f\left(-C_{p}\right)}
$$

where $C_{p}$ is the magnitude of the negative capacitance shunt.

The dashed line in Fig. 5 denotes the impedance of a negative capacitance shunt with the capacitance of -15.5 $\mathrm{nF}$, and it overlaps with the high attenuation zones. This suggest that negative capacitance shunts are highly suitable candidates to attenuate waves for periodic piezoelectric shunt configurations. This result is consistent with Eq. (1) although a different model for the piezoelectric patches was used. Furthermore, based on the current analysis and Fig. 3 to Fig. 5, the attenuation constants for the beams were found to be sensitive to the shunt impedance and the attenuation constant will reduce significantly when the impedance of the shunt deviates from the optimal value. Therefore, for a beam with periodic shunted piezoelectric patches, the ideal strategy is to match the impedance of the shunts to the values in the high attenuation zones across a large range of frequencies. Based on the results from Fig. 5, the optimal type of shunt will be that of a negative capacitance. In order to demonstrate this, the band structure of a periodic beam with piezoelectric patches using an $\mathrm{R}-\mathrm{L}$ shunt and a negative capacitance shunt will be compared.

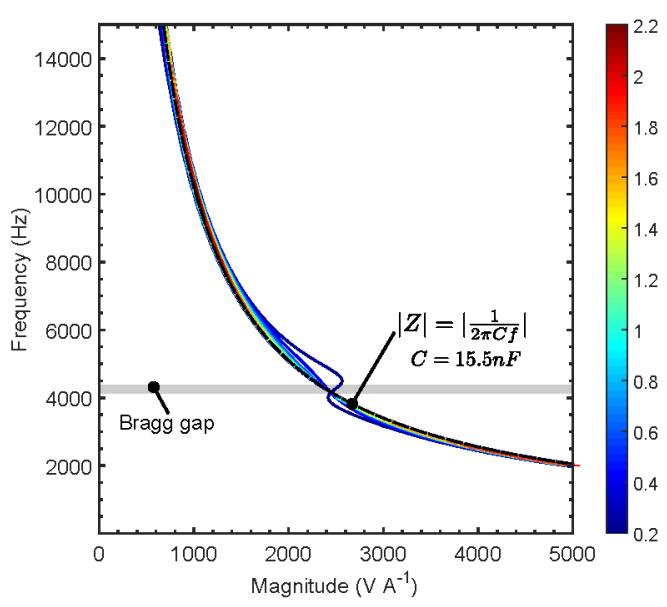

Figure 5. Contour plot of attenuation constant with varying values of $a$ over a range of frequencies at $\phi=\frac{\pi}{2}$.

\section{Comparison between R-L shunt and negative capacitance shunts}

According to the findings in the previous section, the shunting circuit should have impedance with a phase angle of $\frac{\pi}{2} \mathrm{rad}$ and a magnitude within the high attenuation zone shown in Fig. 5 to attenuate elastic waves with band gaps. In order to demonstrate that this is the case irrespective of the type of shunting circuit, the band structure and the transmissibilities of a finite periodic beam with different circuits will be investigated: a resistive-inductive (R-L) circuit, a negative capacitance shunt and a single resistor. These circuits have been considered in previous studies to generate band gaps with periodic piezoelectric patches.

The resistor for the R-L circuit was set to have a low resistance of $0.1 \mathrm{~m} \Omega$, and the inductance was set to be 23.28 $\mathrm{mH}$, to give a resonant frequency of $8000 \mathrm{~Hz}$ for the shunting circuit according to Eq. (27) (Chen et al. (2013b)). For the negative capacitance circuit, a low resistance of $0.1 \mathrm{~m} \Omega$ was set and the capacitance was set to be $-15.5 \mathrm{nF}$ to give the impedance that overlaps with the high attenuation region in Fig. 5. Lastly, the resistor only circuit was set to have a resistance of $1 \Omega$.

$$
f_{n}=\frac{1}{2 \pi \sqrt{L C_{p}}}
$$

where $f_{n}$ is the natural frequency of the shunt and piezoelectric patch circuit and $L$ is the inductance.

Since the R-L and negative capacitance circuits have negligible resistance, the phase angle of the impedance for 
(a)

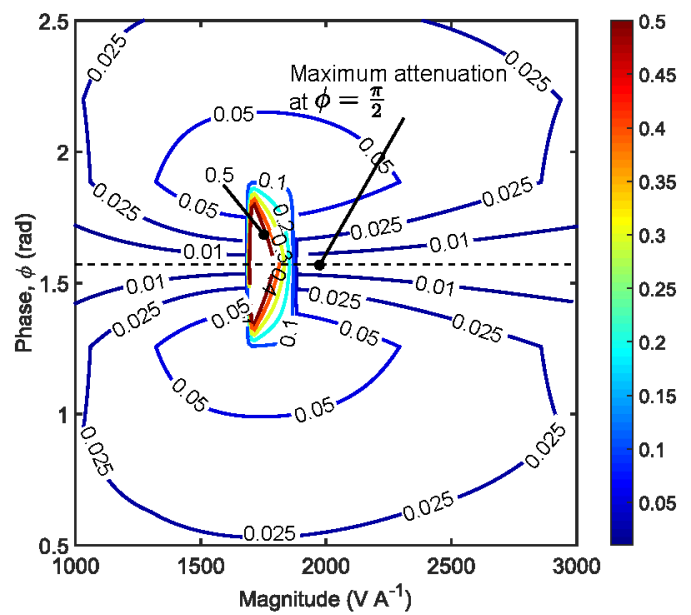

(b)

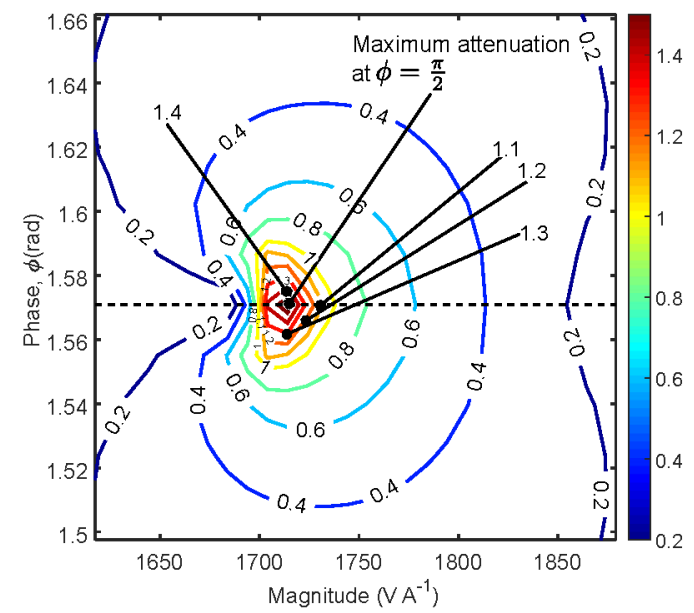

Figure 4. Contour plot of attenuation constant with varying values of $a$ and $\phi$ at $6000 \mathrm{~Hz}$. (a) Results from coarse sweep (b) Results from fine sweep.

the shunting circuits will be close to $\frac{\pi}{2} \mathrm{rad}$, while the phase angle of the impedance of the resistive shunt will always be $0 \mathrm{rad}$. Since the phase angle of the resistive shunt is far from the optimal value of $\frac{\pi}{2}$ rad, it will not produce any band gaps with the exception of the Bragg gap shown in Fig. 3. The band structure of the resistive circuit plotted in Fig. 6 shows that this is the case, where there is only a small band gap at 4151 to $4363 \mathrm{~Hz}$.

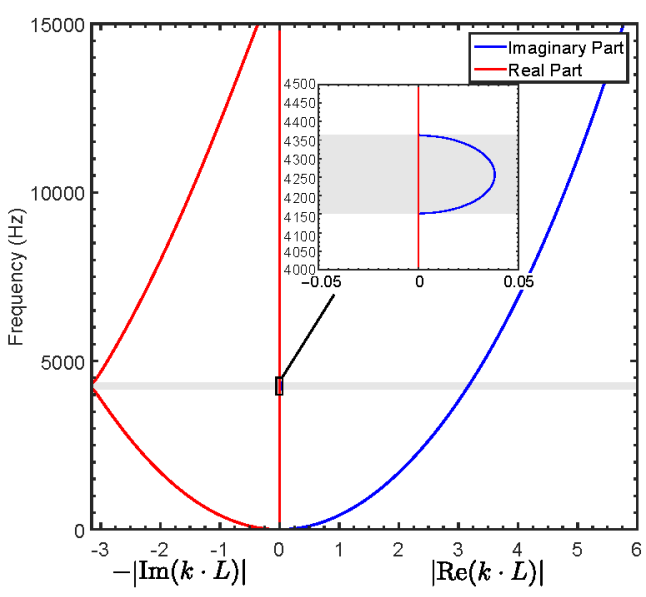

Figure 6. Band Structure of beam with $1 \Omega$ resistor

The magnitudes of the impedance for the R-L and negative capacitance shunts are plotted along with the contours of the attenuation constants in Fig. 7. As seen in Fig. 7, the magnitude of the impedance of the negative capacitance circuit is within the high attenuation zone at frequencies above the Bragg gap and the impedance is on the boundary of the high attenuation zone at frequencies below that. Based on this result, the negative impedance circuit is expected to produce band gaps across the entire frequency range, which is the case as seen in the band structure shown in Fig. 8, where the imaginary part of the wave vectors above 4151
$\mathrm{Hz}$ is large. It is important to note that the imaginary part of the wave vectors at frequencies below $4151 \mathrm{~Hz}$ is very low but not zero, indicating that there is effectively no band gap at the lower frequencies. The negligible values of the imaginary part of the wave vectors at lower frequencies is a result of the impedance being close to the boundary of the high attenuation zone and as discussed in the previous section, the attenuation constants are very sensitive to the impedance and decays rapidly when the impedance deviates from the optimal value which is the case at lower frequencies.

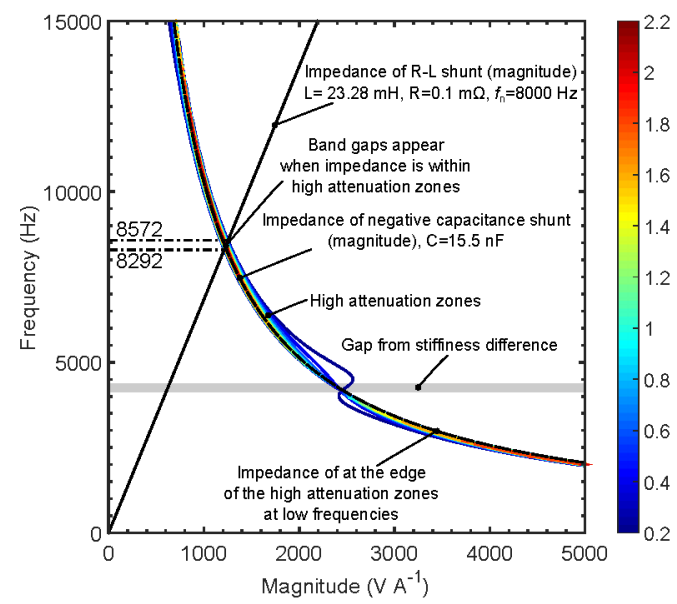

Figure 7. Contour plot of attenuation constants with varying values of $a$ over a range of frequencies at $\phi=\frac{\pi}{2}$ with different circuit configurations.

Furthermore, according to Fig. 7, the magnitude of the impedance of the R-L circuit varies linearly with excitation frequency. The impedance also overlaps with the high attenuation zones at frequencies in between 8292 and 8572 $\mathrm{Hz}$, which is around the tuned resonant frequency of 8000 Hz. In short, the impedance of the R-L circuit will overlap with the high attenuation zone around the tuned frequency. 


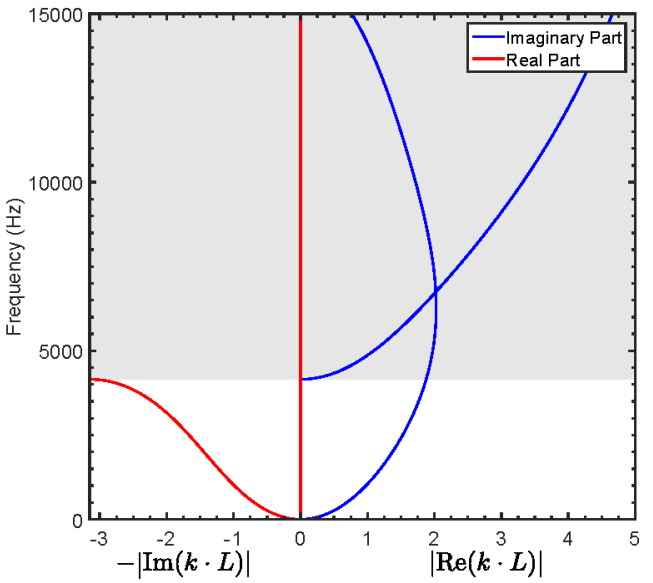

Figure 8. Band structure of beam with negative capacitance shunt.

Therefore, a band gap at this frequency range is expected and this is confirmed by inspecting the band structure of the periodic beam with the R-L shunt in Fig. 9. As seen in Fig. 9 there is a band gap in between frequencies of 8292 and 8572 $\mathrm{Hz}$ and a smaller Bragg gap at 4151 to $4319 \mathrm{~Hz}$.

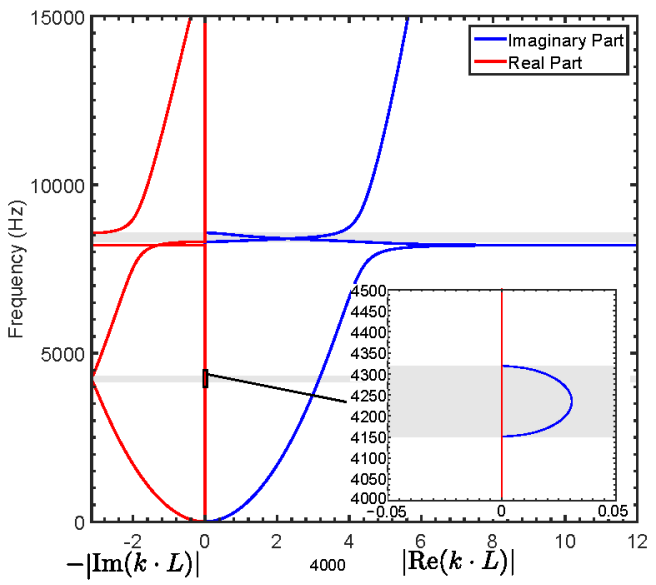

Figure 9. Band Structure of beam with R-L shunt.

The transmissibility curve defined as the magnitude of the ratio of the transverse displacements at the last node to that of the first node, for a finite periodic beam with 10 unit cells is shown in Fig. 10. The values calculated in Fig. 10 is for a free-free beam with a transverse force applied at one end of the beam. As seen in Fig. 10, there are no significant attenuation observed at frequencies where the imaginary wave vectors have very low values for all three circuits, for example the transmissibilities are still high at 4151 to $4363 \mathrm{~Hz}$ although there is a Bragg gap present. However, the transmissibilites are very low for regions where the imaginary wave vectors are large, for example at frequencies above $4151 \mathrm{~Hz}$ for the negative capacitance circuit and at frequencies in between 8292 to $8572 \mathrm{~Hz}$ for the R-L circuit. This result demonstrates the effectiveness of utilising the band gaps produced with the periodic shunted piezoelectric patches to attenuate elastic waves and vibrations.

The key finding from the analysis performed in this section is that effective band gaps will only be present when the phase angle of the shunt impedance is close to $\frac{\pi}{2} \mathrm{rad}$ and the magnitude must overlap with a region of high attenuation constants. Since the only region with high attenuation constant follows a trend where the magnitude decreases exponentially with the frequency, the only method to produce a band gap is to have the shunt impedance be in this region. This applies to all types of shunting circuits, including the R-L circuit that only produces the band gap when its impedance is within the attenuation zone. Therefore, in order to produce band gaps using periodic shunted piezoelectric patches, one should always set the impedance of the shunt to be within the high attenuation zone, which can be estimated by the impedance of a negative capacitance circuit with the magnitude of capacitance being approximately the value of the piezoelectric patch. Furthermore, based on the trends of the impedance of the R-L and negative capacitance shunts, it can be deduced that the negative capacitance shunts are capable of producing large band gaps compared to the R-L shunts as observed in Fig. 7, where the R-L shunt only has a small region of overlap.

Lastly, an important point to note is that the analyses done in this study did not take into account other issues, such as control system instabilities (Beck (2012)), the power consumption of the shunts, the operational limitations of the control system and circuits, and other factors that may be present in the practical implementation of such systems. Hence, care must be taken and some of these factors must be considered before implementing the system in practice. Despite the simplifications made in these analyses, the findings in this study give an overview of the general trend of the attenuation constants across all ranges of passive shunts for beam structures with periodic piezoelectric patches and the shunt design strategy described earlier can serve as a general guide in selecting the optimal shunts.

\section{Summary and conclusion}

Piezoelectric patches arranged periodically across a structure can be used to generate band gaps that are capable of attenuating vibrations when an appropriate shunting circuit is used. Since only two parameters, which are the phase angle, $\phi$, and magnitude, $a$, of the impedance, are required to characterise the entire shunt, the optimal shunt can be found by studying the attenuation constants across a range of frequencies for a beam with periodic piezoelectric patches across an entire range of $\phi$ and $a$.

Based on the volumetric plot showing the attenuation constants for different values of the excitation frequencies, phase angles and magnitudes of the shunt impedance, the optimal impedance was found to have a phase angle that is equal to $\frac{\pi}{2} \mathrm{rad}$ and has a magnitude that decreases exponentially with frequency. The optimal impedance was found to be similar to a negative capacitance shunt with a magnitude of capacitance that is approximately the capacitance of the piezoelectric patch. Additionally, a Bragg gap that is independent of the shunting circuit was found in a frequency range of 4151 to $4363 \mathrm{~Hz}$. Since the attenuation 


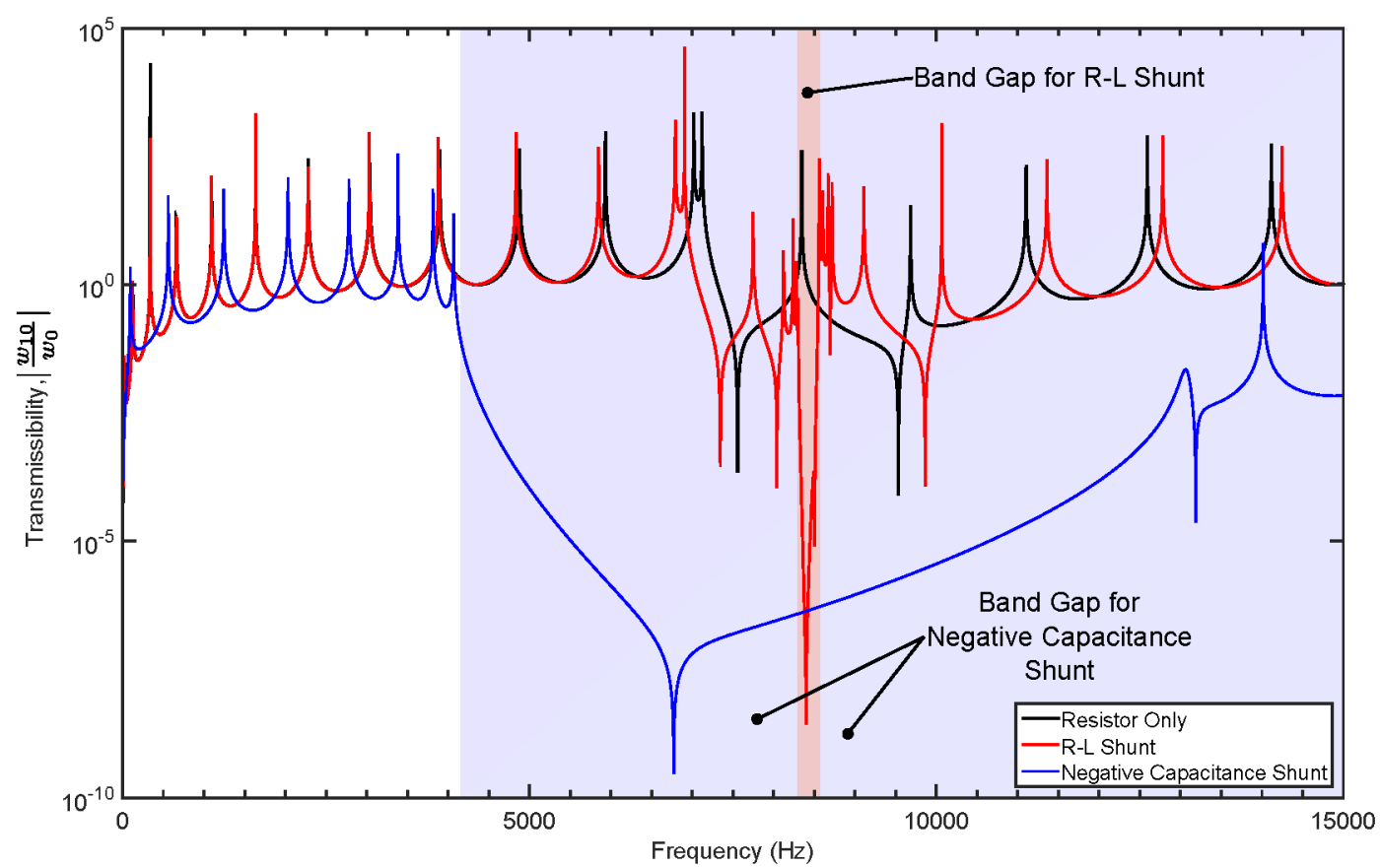

Figure 10. Transmissibility curve for different circuits.

constant is very sensitive to the changes in impedance and reduces significantly when the impedance of the shunt deviates from the optimal value, a negative capacitance shunt will give the largest band gap as it is similar to the trend of the optimal impedance.

The band structures and impedances of several types of circuits, which are the R-L shunt, negative impedance shunt, and resistive shunt, have been studied. The resistive shunt was found to not produce any significant band gap as the phase angle of the resistor will always be constant at 0 rad. Conversely, the R-L and negative capacitance circuits have phase angles of $\frac{\pi}{2} \mathrm{rad}$ as they have negligible resistance. Therefore, these circuits are able to produce band gaps over a range of frequencies.

The negative capacitance shunt was found to produce a large band gap at frequencies above $4151 \mathrm{~Hz}$ as the impedance of the shunt is near the optimal value, while at frequencies below $4151 \mathrm{~Hz}$ the impedance is at the boundary of the attenuation zone and the attenuation constant is negligible in this case. On the other hand, the R-L circuit was found to have impedances within the high attenuation zone around the tuned resonant frequency of $8000 \mathrm{~Hz}$. The band gaps for the R-L circuits only appear within the frequency range where the impedance of the shunt overlaps with the high attenuation zones. This result suggests that regardless of circuit, one should set the impedance of the circuit to the values within the high attenuation zones to attain the most effective elastic wave attenuation with periodic shunted piezoelectric patches. Despite not taking into account some practical limitations, such as control system instabilities, the power consumption of the shunts, etc., the general trends of the attenuation constant with respect to the shunt parameters have been found and a strategy for designing the shunt for periodic piezoelectric patches was devised.

\section{Funding}

The strong support from the Aviation Industry Corporation of China (AVIC), the First Aircraft Institute (FAI) and Beijing Aeronautical Manufacturing Technology Research Institute (BAMTRI) for this funded research is much appreciated. The research was performed at the AVIC Centre for Structural Design and Manufacture at Imperial College London.

\section{Appendix: Terms in the transfer matrix}

The terms in the dynamic stiffness matrix, D, in Eq. (3) are as follows (Ha (2001)).

$\mathbf{Z}_{U}$ is a $2 \times 2$ symmetrical matrix with the non-zero terms defined as follows.

$$
\begin{gathered}
Z_{U}^{(1,1)}=Z_{U}^{(2,2)}=A_{11} \frac{\lambda_{0} \cos \left(\lambda_{0} l\right)}{j \omega \sin \left(\lambda_{0} l\right)} \\
Z_{U}^{(1,2)}=-A_{11} \frac{\lambda_{0}}{j \omega \sin \left(\lambda_{0} l\right)} \\
\lambda_{0}=\omega \sqrt{\frac{\rho_{h}}{A_{11}}} \\
\rho_{h}=\rho_{t} h_{t} b_{t}+\rho_{m} h_{m} b_{m}+\rho_{b} h_{b} b_{b}
\end{gathered}
$$

where $\rho$ is the density, $h$ is the thickness, $b$ is the width and the subscripts $t, m$ and $b$ denote the top, middle and bottom sections of the component.

$$
A_{11}=E_{t}^{E} h_{t} b_{t}+E_{m} h_{m} b_{m}+E_{b}^{E} h_{b} b_{b}
$$

where $E$ is the Young's modulus and the superscript $E$ denotes that the values are evaluated in the short circuit configuration. 
$\mathbf{Z}_{F}$ is a $4 \times 4$ symmetrical matrix with the non-zero terms defined as follows.

$$
\begin{aligned}
& Z_{F}^{(1,1)}=Z_{F}^{(3,3)} \\
& =\left(\frac{1}{X}\right) D_{11} \lambda_{a} \lambda_{b}\left(\lambda_{a}^{2}+\lambda_{b}^{2}\right)\left(\lambda_{b} \lambda_{1}^{2} c_{a} s_{b}-\lambda_{a} \lambda_{2}^{2} c_{b} s_{a}\right) \\
& Z_{F}^{(1,2)}=-Z_{F}^{(3,4)} \\
& =\left(\frac{1}{X}\right) D_{11} \rho_{D}\left[\lambda_{a} \lambda_{b}\left(\lambda_{1}^{2}-\lambda_{2}^{2}\right)\left(c_{a} c_{b}-1\right)\right. \\
& \left.\quad-\left(\lambda_{2}^{2} \lambda_{a}^{2}+\lambda_{1}^{2} \lambda_{b}^{2}\right)\left(s_{a} s_{b}\right)\right] \\
& Z_{F}^{(1,3)}=\left(\frac{1}{X}\right) D_{11} \lambda_{a} \lambda_{b}\left(\lambda_{a}^{2}+\lambda_{b}^{2}\right)\left(\lambda_{a} \lambda_{2}^{2} s_{a}-\lambda_{b} \lambda_{1}^{2} s_{b}\right) \\
& Z_{F}^{(1,4)}=-Z_{F}^{(2,3)} \\
& =-\left(\frac{1}{X}\right) D_{11} \rho_{D} \lambda_{a} \lambda_{b}\left(\lambda_{a}^{2}+\lambda_{b}^{2}\right)\left(c_{a}-c_{b}\right) \\
& Z_{F}^{(2,4)}=\left(\frac{1}{X}\right) D_{11} \rho_{D}\left(\lambda_{a}^{2}+\lambda_{b}^{2}\right)\left(\lambda_{a} \lambda_{2}^{2} s_{b}+\lambda_{b} \lambda_{1}^{2} s_{a}\right) \\
& Z_{F}^{(2,2)}=Z_{F}^{(4,4)} \\
& \left(\frac{1}{X}\right) D_{11} \rho_{D}\left(\lambda_{a}^{2}+\lambda_{b}^{2}\right)\left(\lambda_{a} \lambda_{2}^{2} c_{a} s_{b}-\lambda_{b} \lambda_{1}^{2} c_{b} s_{a}\right)
\end{aligned}
$$

$$
X=j \omega\left[2 \rho_{D} \lambda_{a} \lambda_{b}\left(c_{a} c_{b}-1\right)+s_{a} s_{b}\left(\lambda_{1}^{4} \lambda_{b}^{2}-\lambda_{2}^{4} \lambda_{a}^{2}\right)\right]
$$

$$
s_{a}=\sin \left(\lambda_{a} l\right)
$$$$
c_{b}=\cosh \left(\lambda_{b} l\right)
$$$$
s_{b}=\sinh \left(\lambda_{b} l\right)
$$$$
\lambda_{1}^{2}=\lambda_{a}^{2}-\rho_{S}
$$$$
\lambda_{2}^{2}=\lambda_{b}^{2}+\rho_{S}
$$

$$
\lambda_{a}=\sqrt{\frac{1}{2}\left(\rho_{S}+\rho_{R}\right)+\sqrt{\frac{1}{4}\left(\rho_{S}-\rho_{R}\right)^{2}+\rho_{D}}}
$$

$$
\lambda_{b}=\sqrt{-\frac{1}{2}\left(\rho_{S}+\rho_{R}\right)+\sqrt{\frac{1}{4}\left(\rho_{S}-\rho_{R}\right)^{2}+\rho_{D}}}
$$

$$
\begin{gathered}
\rho_{S}=\frac{\rho_{h} \omega^{2}}{A_{55}} \\
\rho_{R}=\frac{\rho_{r} \omega^{2}}{D_{11}} \\
\rho_{D}=\frac{\rho_{h} \omega^{2}}{D_{11}} \\
\rho_{r}=\frac{\rho_{t} b_{t}}{3}\left[\left(\frac{h_{m}}{2}+h_{t}\right)^{3}-\left(\frac{h_{m}}{2}\right)^{3}\right]+\frac{\rho_{m} b_{m}}{12} h_{m}^{3}+ \\
\frac{\rho_{b} b_{b}}{3}\left[\left(\frac{h_{m}}{2}+h_{b}\right)^{3}-\left(\frac{h_{m}}{2}\right)^{3}\right]
\end{gathered}
$$

where $\rho$ are the densities.

$$
\begin{gathered}
D_{11}=\frac{E_{t}^{E} b_{t}}{3}\left[\left(\frac{h_{m}}{2}+h_{t}\right)^{3}-\left(\frac{h_{m}}{2}\right)^{3}\right]+\frac{E_{m} b_{m}}{12} h_{m}^{3}+ \\
\frac{E_{b}^{E} b_{b}}{3}\left[\left(\frac{h_{m}}{2}+h_{b}\right)^{3}-\left(\frac{h_{m}}{2}\right)^{3}\right] \\
A_{55}=\kappa\left(G_{t}^{E} b_{t} h_{t}+G_{m}^{E} b_{m} h_{m}+G_{b}^{E} b_{b} h_{b}\right)
\end{gathered}
$$

The electromechanical coupling matrices, $\mathbf{P}_{N}$ and $\mathbf{P}_{F}$, are defined as follows.

$$
\mathbf{P}_{N}=\left[\begin{array}{cc}
d_{31, t} E_{t}^{E} & d_{31, b} E_{b}^{E} \\
-d_{31, t} E_{t}^{E} & -d_{31, b} E_{b}^{E}
\end{array}\right]\left[\begin{array}{l}
b_{t} \\
b_{b}
\end{array}\right]
$$

where $d_{31}$ is the piezoelectric voltage coefficient.

$$
\mathbf{P}_{F}=\left[\begin{array}{cc}
-d_{31, t} E_{t}^{E} & -d_{31, b} E_{b}^{E} \\
0 & 0 \\
d_{31, t} E_{t}^{E} & d_{31, b} E_{b}^{E} \\
0 & 0
\end{array}\right]\left[\begin{array}{l}
b_{t} z_{t} \\
b_{t} z_{b}
\end{array}\right]
$$

The clamped admittance matrix, $\mathbf{C}$, is defined as follows.

$$
\mathbf{C}=i \omega l\left[\begin{array}{cc}
\epsilon_{33, t}^{T}-d_{31, t}^{2} E_{t}^{E} & 0 \\
0 & \epsilon_{33, b}^{T}-d_{31, b}^{2} E_{b}^{E}
\end{array}\right]\left[\begin{array}{l}
\frac{b_{t}}{h_{t}} \\
\frac{b_{b}}{h_{b}}
\end{array}\right]
$$

\section{References}

Airoldi L and Ruzzene M (2011) Design of tunable acoustic metamaterials through periodic arrays of resonant shunted piezos. New Journal of Physics 13(11): 113010.

Beck BS (2012) Negative capacitance shunting of piezoelectric patches for vibration control of continous systems .

Beck BS, Cunefare Ka, Collet M and Ruzzene M (2012) New Method of Negative Capacitance Shunt Tuning for Vibration Control 8341: 834106-834106-11.

Behrens S, Moheimani S and aJ Fleming (2003) Multiple mode current flowing passive piezoelectric shunt controller. Journal of Sound and Vibration 266(5): 929-942.

Brillouin L (1946) Wave Propagation In Periodic Structures. McGraw-Hill Book Company. 
1

2

3

Casadei F, Beck BS, Cunefare Ka and Ruzzene M (2012) Vibration control of plates through hybrid configurations of periodic piezoelectric shunts. Journal of Intelligent Material Systems and Structures 23(10): 1169-1177.

Chen S, Wang G, Wen J and Wen X (2013a) Wave propagation and attenuation in plates with periodic arrays of shunted piezopatches. Journal of Sound and Vibration 332(6): 1520-1532.

Chen SB, Wen JH, Wang G and Wen XS (2013b) Tunable band gaps in acoustic metamaterials with periodic arrays of resonant shunted piezos. Chinese Physics B 22(7): 074301.

Chen SB, Wen JH, Yu DL, Wang G and Wen XS (2011) Band gap control of phononic beam with negative capacitance piezoelectric shunt. Chinese Physics B 20(1): 014301.

Ha SK (2001) Analysis of the asymmetric triple-layered piezoelectric bimorph using equivalent circuit models. The Journal of the Acoustical Society of America 110(2): 856.

Hagood N and von Flotow A (1991) Damping of structural vibrations with piezoelectric materials and passive electrical networks. Journal of Sound and Vibration 146: 243-268.

Hussein MI, Leamy MJ and Ruzzene M (2014) Dynamics of Phononic Materials and Structures: Historical Origins, Recent Progress, and Future Outlook. Applied Mechanics Reviews 66(4): 040802.

Liu Z, Zhang X, Mao Y, Zhu Y, Yang Z, Chan C and Ping S (2000) Locally Resonant Sonic Materials. Science 289: 1734-1736.

Mathworks (2014) Matlab Documentaion.

PI Instruments (2014) Piezoelectric Ceramic Products: Fundamentals, characteristics and applications.

Spadoni A, Ruzzene M and Cunefare K (2009) Vibration and Wave Propagation Control of Plates with Periodic Arrays of Shunted Piezoelectric Patches. Journal of Intelligent Material Systems and Structures 20(8): 979-990.

Tateo F, Beck BS, Collet M, Ouisse M, Cunefare Ka and Ichchou MN (2014) Vibration control of plates through a periodic array of shunted piezoelectric patches with negative capacitance circuits 9057: 90572E.

Thorp O, Ruzzene M and Baz A (2001) Attenuation and localization of wave propagation in rods with periodic shunted piezoelectric patches. Smart Materials and Structures 979.

Wu Sy and McDonnell (1996) Piezoeleciric shunts with a parallel R-L circuit for structural damping and vibration control. Smart Structures and Materials 1996: Passive Damping and Isolation 27201259(Lc). 


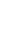
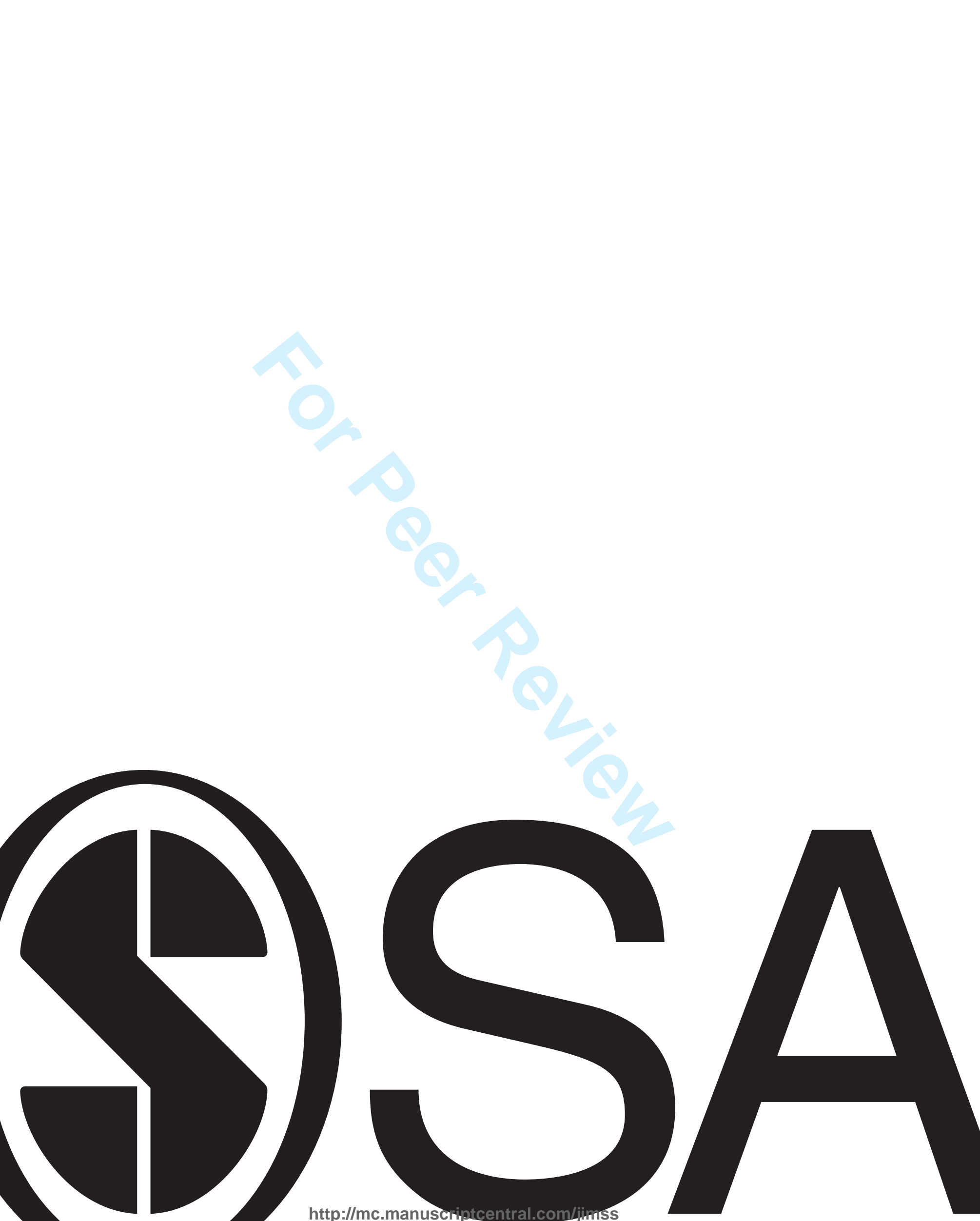

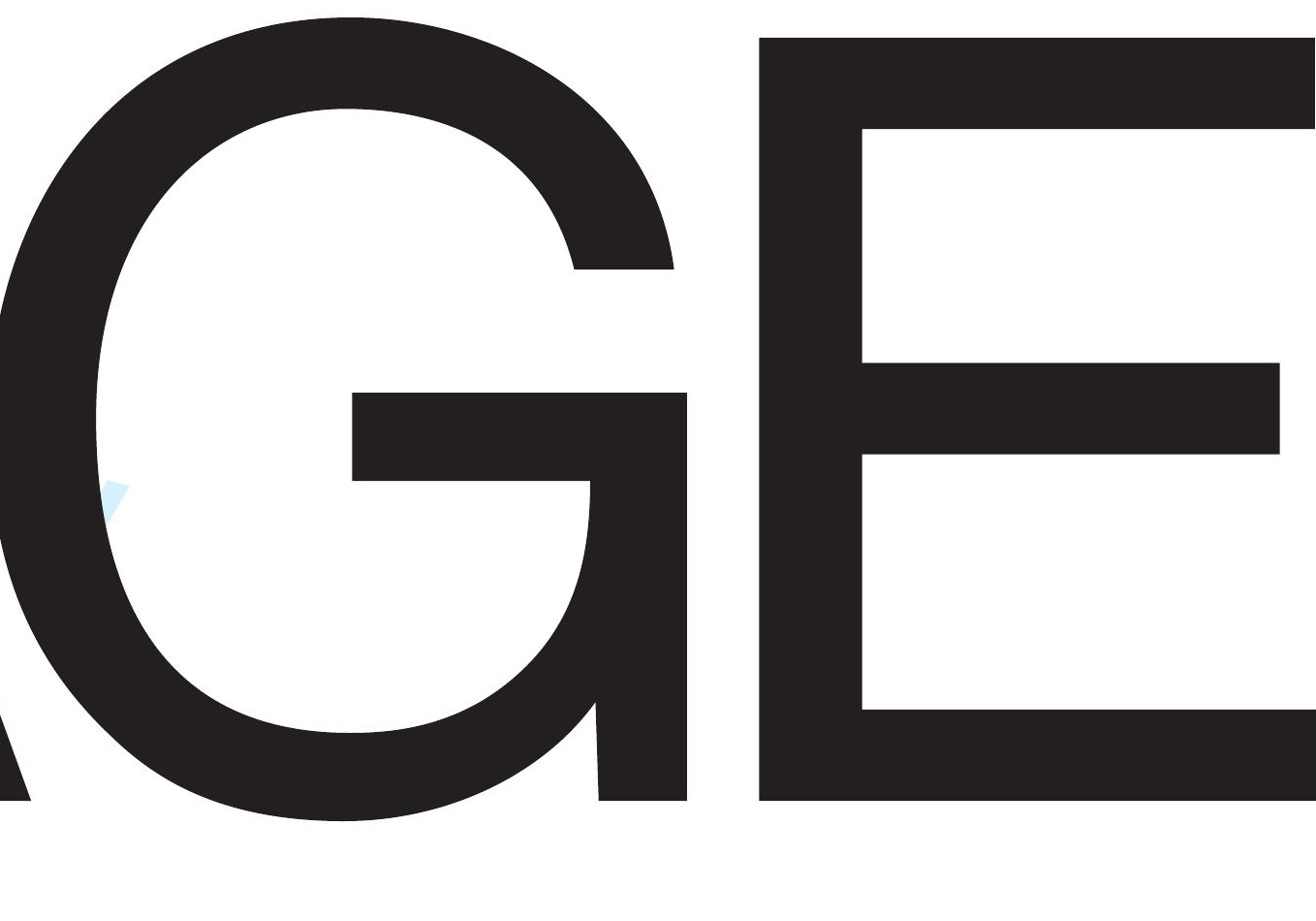